\title{
Overview of SCIAMACHY validation: 2002-2004
}

\author{
A. J. M. Piters ${ }^{1}$, K. Bramstedt ${ }^{2}$, J.-C. Lambert ${ }^{3}$, and B. Kirchhoff ${ }^{4,5}$ \\ ${ }^{1}$ Royal Netherlands Meteorological Institute, Department of Climate Research and Seismology, De Bilt, The Netherlands \\ ${ }^{2}$ Institute for Environmental Physics, University of Bremen, Germany \\ ${ }^{3}$ Belgian Institute for Space Aeronomy, Brussels, Belgium \\ ${ }^{4}$ Institute for Environmental Physics, University of Heidelberg, Germany \\ ${ }^{5}$ Max Planck Institute for Chemistry, Department for Atmospheric Chemistry, Mainz, Germany
}

Received: 11 April 2005 - Published in Atmos. Chem. Phys. Discuss.: 30 August 2005

Revised: 20 December 2005 - Accepted: 20 December 2005 - Published: 25 January 2006

\begin{abstract}
SCIAMACHY, on board Envisat, has been in operation now for almost three years. This UV/visible/NIR spectrometer measures the solar irradiance, the earthshine radiance scattered at nadir and from the limb, and the attenuation of solar radiation by the atmosphere during sunrise and sunset, from 240 to $2380 \mathrm{~nm}$ and at moderate spectral resolution. Vertical columns and profiles of a variety of atmospheric constituents are inferred from the SCIAMACHY radiometric measurements by dedicated retrieval algorithms. With the support of ESA and several international partners, a methodical SCIAMACHY validation programme has been developed jointly by Germany, the Netherlands and Belgium (the three instrument providing countries) to face complex requirements in terms of measured species, altitude range, spatial and temporal scales, geophysical states and intended scientific applications. This summary paper describes the approach adopted to address those requirements.

Since provisional releases of limited data sets in summer 2002, operational SCIAMACHY processors established at DLR on behalf of ESA were upgraded regularly and some data products - level-1b spectra, level- $2 \mathrm{O}_{3}, \mathrm{NO}_{2}, \mathrm{BrO}$ and clouds data - have improved significantly. Validation results summarised in this paper and also reported in this special issue conclude that for limited periods and geographical domains they can already be used for atmospheric research. Nevertheless, current processor versions still experience known limitations that hamper scientific usability in other periods and domains. Free from the constraints of operational processing, seven scientific institutes (BIRA-IASB, IFE/IUP-Bremen, IUP-Heidelberg, KNMI, MPI, SAO and SRON) have developed their own retrieval algorithms and generated SCIAMACHY data products, together addressing nearly all targeted constituents. Most of the UV-visible data products $-\mathrm{O}_{3}, \mathrm{NO}_{2}, \mathrm{SO}_{2}, \mathrm{H}_{2} \mathrm{O}$ total columns; $\mathrm{BrO}, \mathrm{OClO}$
\end{abstract}

Correspondence to: A. J. M. Piters

(piters@knmi.nl) slant columns; $\mathrm{O}_{3}, \mathrm{NO}_{2}, \mathrm{BrO}$ profiles - already have acceptable, if not excellent, quality. Provisional near-infrared column products - $\mathrm{CO}, \mathrm{CH}_{4}, \mathrm{~N}_{2} \mathrm{O}$ and $\mathrm{CO}_{2}$ - have already demonstrated their potential for a variety of applications. Cloud and aerosol parameters are retrieved, suffering from calibration with the exception of cloud cover. In any case, scientific users are advised to read carefully validation reports before using the data. It is required and anticipated that SCIAMACHY validation will continue throughout instrument lifetime and beyond and will accompany regular processor upgrades.

\section{Introduction}

SCIAMACHY (SCanning Imaging Absorption spectroMeter for Atmospheric CartograpHY) is a spectrometer on-board ESA's Environmental Satellite Envisat launched on 1 March 2002. SCIAMACHY measures sunlight reflected or transmitted by the Earth's atmosphere in the ultraviolet, visible, and near-infrared wavelength region $(240-2380 \mathrm{~nm})$ at moderate spectral resolution. SCIAMACHY observes earthshine radiance in limb and nadir viewing geometry and solar and lunar light transmitted through the atmosphere in occultation viewing geometry.

The primary objective of the SCIAMACHY mission is to improve our knowledge of the chemistry and physics of the Earth's atmosphere (troposphere, stratosphere, and mesosphere), including anthropogenic changes or the variability of natural phenomena. SCIAMACHY's contribution are vertical and horizontal distributions of atmospheric constituents and parameters such as trace gases, aerosols, cloud information, temperature and pressure. Specific topics for SCIAMACHY's scientific mission are:

- stratospheric ozone chemistry, including the verification of expected effects of the Montreal Protocol and

(C) 2006 Author(s). This work is licensed under a Creative Commons License. 


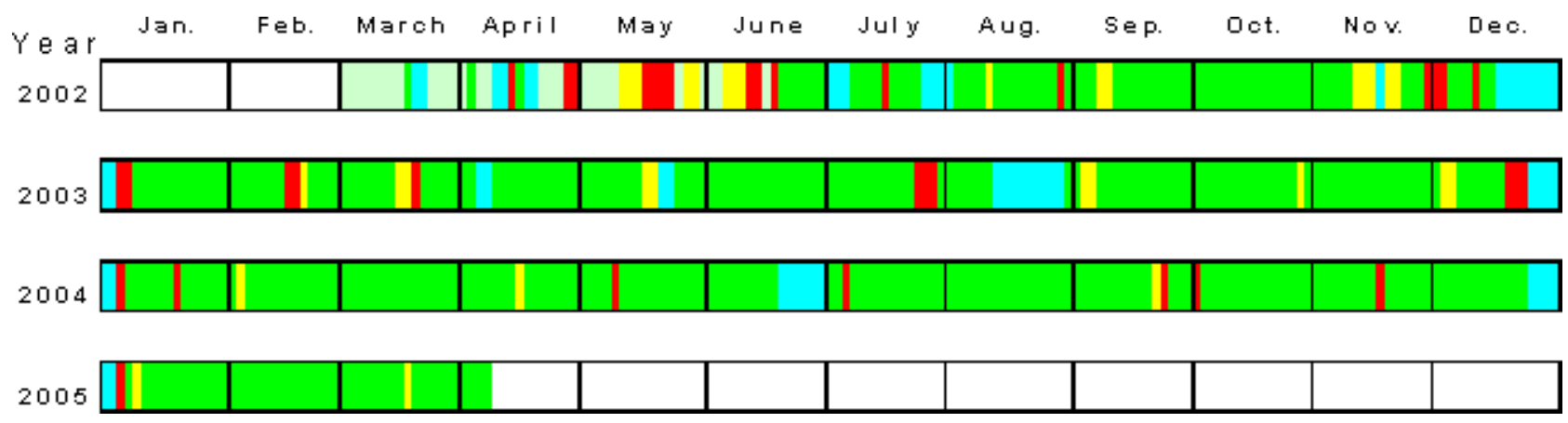

Fig. 1. Overview of SCIAMACHY operations between March 2002 and April 2005. Green denotes periods with nominal measurements, blue are the periods where the instrument was heated to evaporate the ice layer on the detectors (the near infra-red channels cannot be used during this period for retrieval of $\mathrm{CO}, \mathrm{CH}_{4}, \mathrm{~N}_{2} \mathrm{O}, \mathrm{CO}_{2}$ ), yellow are periods where Envisat was off, red are the periods where SCIAMACHY was off. Courtesy: C. Chlebek, DLR.

Table 1. Availability of the anticipated level 2 products from SCIAMACHY. Note that some of the "recommended" products are in fact included in the operational data files, but they have no geophysical meaning yet. "NRT": near-real time; "OL": offline (see Sect. 5); "av": available; "glob": larger or global dataset; "rec": recommended, not available; "cs": few case study periods; "dev": under development; "-": not planned or development not started.

\begin{tabular}{|c|c|c|c|c|}
\hline \multirow[t]{2}{*}{ Product } & \multirow[t]{2}{*}{ spectral region } & \multicolumn{2}{|c|}{ operational } & \multirow[t]{2}{*}{ scientific } \\
\hline & & NRT & $\mathrm{OL}$ & \\
\hline $\mathrm{O}_{3}$ column & $\mathrm{UV} / \mathrm{vis}$ & av & av & glob \\
\hline $\mathrm{NO}_{2}$ column & $\mathrm{UV} /$ vis & av & av & glob \\
\hline $\mathrm{BrO}$ column & $\mathrm{UV} / \mathrm{vis}$ & av & av & glob \\
\hline $\mathrm{SO}_{2}$ column & UV/vis & rec & rec & glob \\
\hline OClO column & $\mathrm{UV} / \mathrm{vis}$ & rec & rec & glob \\
\hline HCHO column & $\mathrm{UV} /$ vis & rec & rec & dev \\
\hline $\mathrm{H}_{2} \mathrm{O}$ column & UV/vis,NIR & rec & rec & cs \\
\hline $\mathrm{N}_{2} \mathrm{O}$ column & UV/vis & rec & rec & cs \\
\hline CO column & $\mathrm{UV} / \mathrm{vis}$ & rec & rec & cs \\
\hline $\mathrm{CO}_{2}$ column & $\mathrm{UV} / \mathrm{vis}$ & rec & rec & cs \\
\hline $\mathrm{CH}_{4}$ column & $\mathrm{UV} / \mathrm{vis}$ & rec & rec & cs \\
\hline cloud cover & $\mathrm{UV} /$ vis & av & av & glob \\
\hline cloud top height & $\mathrm{UV} /$ vis & rec & rec & glob \\
\hline AAI & UV/vis,NIR & rec & rec & cs \\
\hline AOT & UV/vis,NIR & rec & rec & cs \\
\hline $\mathrm{O}_{3}$ profile & UV/vis & - & av & glob \\
\hline $\mathrm{NO}_{2}$ profile & $\mathrm{UV} /$ vis & - & av & glob \\
\hline $\mathrm{BrO}$ profile & UV/vis & - & rec & glob \\
\hline OClO profile & $\mathrm{UV} / \mathrm{vis}$ & - & rec & cs \\
\hline $\mathrm{H}_{2} \mathrm{O}$ profile & UV/vis,NIR & - & rec & dev \\
\hline $\mathrm{CO}_{2}$ profile & NIR & - & rec & - \\
\hline $\mathrm{CH}_{4}$ profile & NIR & - & rec & - \\
\hline $\mathrm{N}_{2} \mathrm{O}$ profile & NIR & - & rec & - \\
\hline CO profile & NIR & - & rec & - \\
\hline pressure profile & UV/vis,NIR & - & rec & - \\
\hline temperature profile & UV/vis,NIR & - & rec & - \\
\hline aerosol profile & UV/vis,NIR & - & rec & - \\
\hline
\end{tabular}

Atmos. Chem. Phys., 6, 127-148, 2006 amendments on future developments of the ozone layer;

- climate research by observation of radiatively active species and transport tracers important for understanding global warming, like greenhouse gases, aerosols, and clouds;

- tropospheric pollution associated with industrial activity, urban concentration and biomass burning;

- troposphere-stratosphere exchange processes;

- monitoring and understanding of special events and hazards such as volcanic eruptions, solar proton events, and related regional and global consequences.

A complete description of SCIAMACHY and its mission can be found in Bovensmann et al. (1999) and references therein.

SCIAMACHY has been in operation for more than three years now, and is a very stable instrument with only few short periods of "off-time" as was shown by Chlebek et al. (2004), see Fig. 1. The detectors are regularly heated up (decontamination) to evaporate a small ice layer on the NIR detectors.

A number of instrumental and calibration issues are known to affect the quality of the retrieved trace gases. The issues which are currently (April 2005) believed to affect the retrievals most are (Lichtenberg et al., 2005): 1) a radiance offset and a different irradiance offset, 2) insufficient polarisation correction, 3) errors in calibration of NIR detectors (channel 7 light leak, changing "slit function", thermal contribution to dark current).

The currently retrieved SCIAMACHY level 2 data products are listed in Table 1. The main objective of the validation is to accompany each of these products by a complete description of the systematic and random deviations from other well-established measurement systems, formulated in such a way that it is of direct use for algorithm improvement, instrument characterisation and atmospheric research. 
To achieve this, we need: (1) a large amount of suitable validation measurements and (2) detailed analyses of the SCIAMACHY data with respect to the validation data set. This is more than a straightforward calculation of the average bias and scatter, as is outlined in Sect. 2, where the validation methods are described.

In Sect. 3 an overview is presented of the overall organisation of the international validation of SCIAMACHY. Section 4 describes the validation measurements performed in dedicated campaigns and by regular measurement systems, often organised in global networks. The contents, availability and expected upgrades of the SCIAMACHY products are described in Sect. 5. Preliminary validation results are summarised in Sect. 6, and the necessary future validation efforts will be discussed in Sect. 7.

\section{Validation methods}

Satellite validation is often understood as a simple comparison exercise concluding to a once-and-for-all assessment of the difference between the satellite data being validated and a reference data set of "validated" quality. Although such comparisons are indeed the basis for investigating the quality of the satellite data, they are by no means sufficient for assessing the usefulness of the data for its intended scientific applications. Finding an agreement within estimated error bars offers no guarantee that the retrieved values contain new information coming from the measurement itself. Therefore, beyond the calculation of differences between SCIAMACHY and correlative data sets, it is recommended to use several other validation methods, each with its own potential contribution to the overall assessment of the usefulness of the data.

It is important to investigate, both qualitatively and quantitatively, how well SCIAMACHY data represents known geophysical signals that are either observed by other measurement systems or deduced from our understanding of the atmosphere. These signals may include meridional and zonal structures, vertical structures, temporal cycles on seasonal, day-to-day and diurnal scales, special events of tropospheric pollution, etc.

Geophysical (level 2) quantities are retrieved from SCIAMACHY spectra (level 1) using auxiliary data, such as output from radiative transfer models, or climatologies. Simplifications or misinterpretations therein can result in systematic errors in the retrieved quantities that may depend on geophysical, instrumental or algorithm parameters. It is important to investigate the influence of these parameter-dependent systematic errors on the intended scientific use.

Validation also plays a diagnostic role in the improvement of retrieval algorithms. Careful investigation of comparison time-series, the use of assimilation tools, and the intercomparison of SCIAMACHY data retrieved with independent algorithms have been powerful in revealing internal inconsistencies in SCIAMACHY data.
The comparison of remotely sensed geophysical quantities with correlative data is not straightforward. A major difficulty results from the convolution of atmospheric variability with the smoothing/scanning properties inherent to the remote sensing approach. Different observation techniques and retrieval methods yield different sampling of the atmosphere in time and in space, different averaging of its 3-dimensional structure, and different sensitivity to ancillary atmospheric and instrumental parameters. As a direct consequence of those differences in the perception of the atmospheric field, atmospheric structures and variability can critically corrupt the reliability of the comparison by introducing systematic biases and additional scatter. Therefore, sophisticated methods have been developed to deal with representativeness errors in the comparisons:

- the use of radiative transfer tools to better determine the vertical and line-of-sight smoothing of both SCIAMACHY and correlative data (modelling of slant column, weighting functions, averaging kernels);

- the use of modelling and assimilation tools to deal with transport and photochemical effects (including diurnal cycles);

- the use of meteorological analyses to discriminate the effects of dynamic variability (e.g., use of backward trajectories, transformation to equivalent latitude and isentropic coordinates);

- the synergistic use of complementary correlative data sources offering different smoothing/sampling properties, sensitivity and errors budgets.

The latter point is of great importance for SCIAMACHY validation. The SCIAMACHY data products potentially support an assortment of scientific applications, spanning from regional to global scales, from the ground up to the mesosphere, from short-term to decadal timeframes. Local studies carried out at single stations constitute the preferred approach to detailed investigation. They benefit from local research and excellent understanding of local geophysical particulars; they assure full control and accurate error budgets of the instrumentation, and the availability of adequate ancillary data. Complementary studies exploiting pseudo-global sources yield access to patterns, sensitivity and space/time structures on the global scale. Satellite-satellite intercomparisons improve the statistical significance of validation results due to the large amount of possible collocations.

\section{Validation organisation}

SCIAMACHY is an Announcement of Opportunity (AO) instrument provided by the AO instrument Providers (AOIPs) Germany and the Netherlands with a contribution from Belgium. In principle, the AOIPs have the responsibility for the 


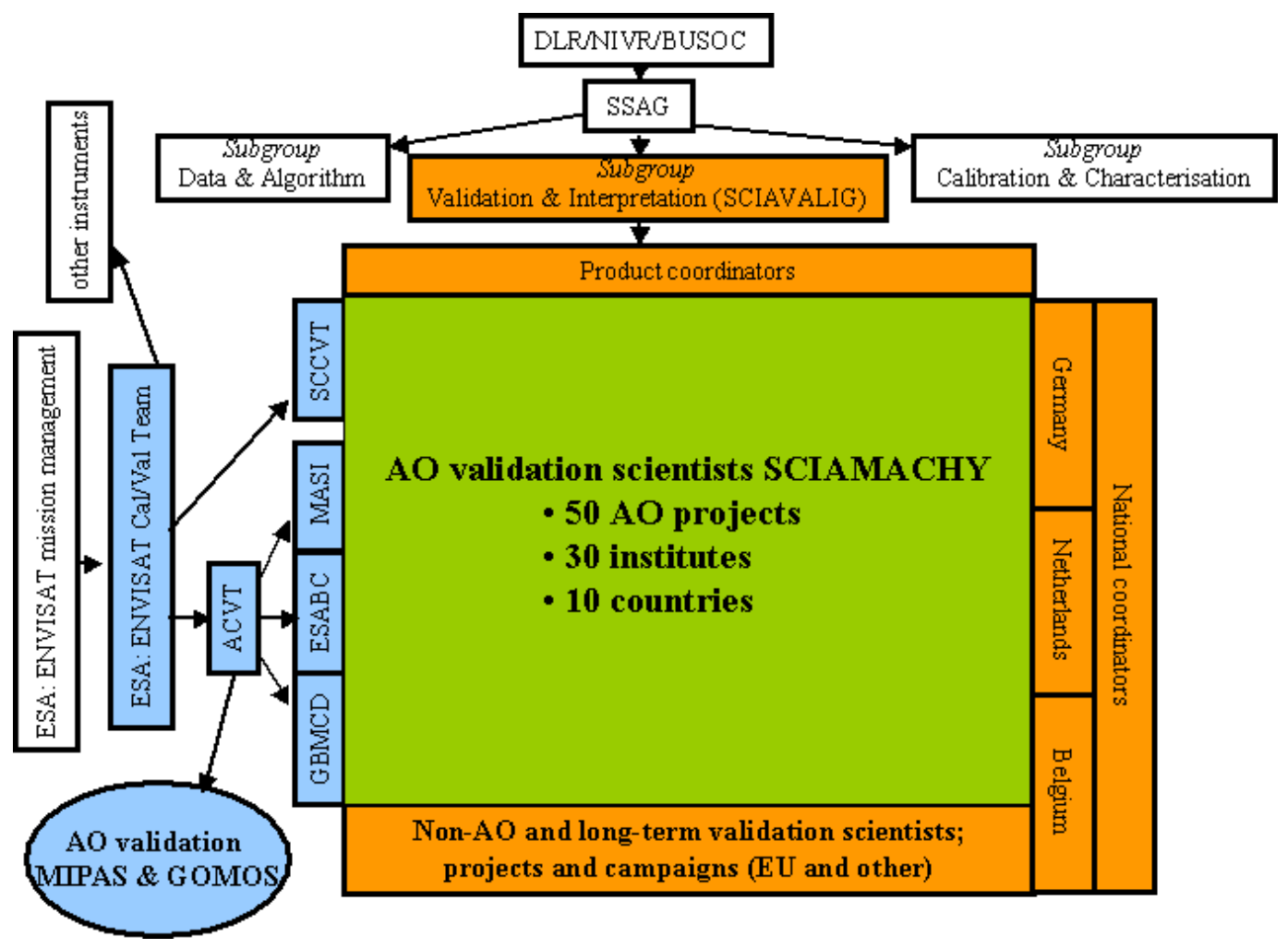

Fig. 2. A scheme of the SCIAMACHY validation organisational structures set-up by SCIAVALIG (orange) and by ESA (blue). The validation scientists actually doing the work are supported by both organisations, if they have an approved AO proposal for SCIAMACHY validation (green).

validation of SCIAMACHY, but since ESA has the responsibility for the operational SCIAMACHY data processor, ESA includes the validation of SCIAMACHY into their Envisat validation programme as well. The two complementary organisation structures from ESA and SCIAVALIG are illustrated in Fig. 2.

\subsection{ESA's organisation}

In 1997 ESA raised an Announcement of Opportunity (AO) for the use of Envisat data. The Principal Investigators of the approved AO projects dealing with the validation of SCIAMACHY, GOMOS and MIPAS were gathered in the Atmospheric Chemistry Validation Team (ACVT). The ACVT is divided in the following subgroups:

GBMCD: Ground-based Measurements and Campaign Database subgroup,

ESABC: Envisat Stratospheric Aircraft and Balloon Campaign, and

MASI: Models and data Assimilation, Satellite Intercomparisons.

A subgroup of the overall Envisat Calibration and Validation team is also the:
SCCVT: SCIAMACHY Calibration and Verification Team.

The ESABC subgroup is more than a working group. ESA, DLR, and CNES together financed dedicated campaigns for the validation of SCIAMACHY, MIPAS and GOMOS (Sect. 4.3). These campaigns are referred to as ESABC campaigns, and are prepared and coordinated in the ESABC group. Preparation and results of other campaigns are only presented and discussed within in the ESABC group.

In addition, ESA supports a dedicated validation data centre, the NILU Atmospheric Data Base for Interactive Retrieval (NADIR), which is operated by the Norwegian Institute for Air Research (NILU). This centre hosts all correlative measurements from the dedicated Envisat validation campaigns.

\subsection{SCIAVALIG's organisation}

The organisation of the validation of SCIAMACHY from the AOIP's side is delegated to the SCIAMACHY VALidation and Interpretation Group (SCIAVALIG), a subgroup of the SCIAMACHY Science Advisory Group (SSAG). SCIAVALIG consists of an international scientific consortium of representatives of 12 institutes participating in the 
validation. It is chaired by the Royal Netherlands Meteorological Institute (KNMI), the Belgian Institute for Space Aeronomy (BIRA-IASB), and the University of Heidelberg (IUP-Heidelberg). SCIAVALIG has established a list of validation requirements (SCIAVALIG, 1998), defined an essential "core" validation programme (SCIAVALIG, 2002), and has set up an organisational structure for the continuous monitoring of validation results throughout the lifetime of SCIAMACHY. The core validation programme is mainly funded by the AOIPs, and is embedded in the ESA AO programme via several AO projects. The "national coordinators" of SCIAVALIG (who are the authors of this paper) are responsible for the practical coordination of this programme.

To take the best benefit of every component of the complementary efforts and structures, "product coordinators" have been appointed by SCIAVALIG to collect and digest validation results from the different contributors, address apparent inconsistencies, identify unsolved needs, and foster interaction with algorithm developing teams and processor experts for an efficient translation from validation results towards algorithm changes.

\section{Correlative measurements}

The core validation programme was complemented by a selection of $\mathrm{AO}$ projects from international partners. The major component of the SCIAMACHY validation programme consists of comparison studies with correlative measurements acquired by independent instrumentations from various platforms, namely, ground-based stations and ships, aircrafts, stratospheric balloons, and satellites.

\subsection{Ground-based and ship-based instruments}

Ground-based instruments provide the appropriate correlative data to fulfil four main tasks of the SCIAMACHY validation programme:

- quick validation before public release of a new product or just after the release of a near-real time product;

- detailed geophysical validation from pole to pole and for a variety of geophysical states, including dependences on measurement and atmospheric parameters like, e.g., solar zenith angle and temperature;

- after major improvement of a retrieval algorithm, verification of correctness of changes and preliminary quality assessment of the resulting data product;

- long-term validation, including detection of trends and other time-varying features.

The list of stations providing correlative measurements for SCIAMACHY validation is given in Table 2 . The nationally funded core validation programme, constituting the backbone of the validation, includes complementary types of instrumentation (see list in the next paragraph), yielding all

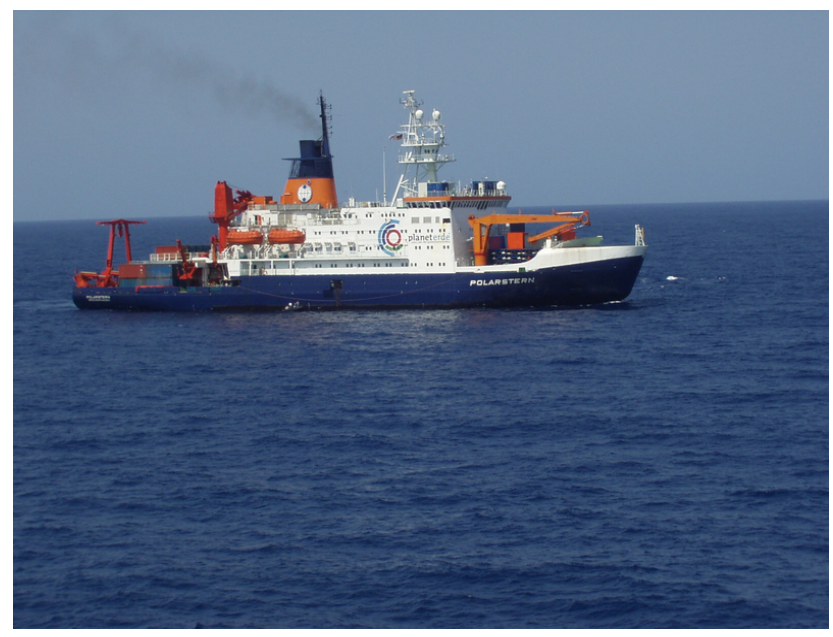

Fig. 3. The German Research vessel Polarstern.

together nearly all targeted species, and operating at about forty stations distributed from the Arctic to the Antarctic and from South America to the Indian Ocean, as indicated in Table 2. Thanks to long-lasting collaborations established mainly in the framework of WMO's Global Atmospheric Watch programme (GAW), and particularly within its affiliated ozonometric networks (see Fioletov et al., 1999, and references therein) and the Network for the Detection of Stratospheric Change (NDSC, Lambert et al., 1999, and references therein), international partners contribute through AO projects with a long list of instruments which add significantly to the geographical coverage of the ground-based instrumentation included in the core validation programme.

The ozone column amount is monitored at a variety of ground-based stations by Dobson and Brewer ultraviolet spectrophotometers and by Russian/NIS ultraviolet filter radiometers of the M-124 design. A network of about 30 DOAS instruments, all certified for the NDSC, monitor the column amount of species absorbing in the UV-visible part of the spectrum like $\mathrm{O}_{3}, \mathrm{NO}_{2}, \mathrm{BrO}, \mathrm{OClO}, \mathrm{HCHO}, \mathrm{SO}_{2}$, $\mathrm{H}_{2} \mathrm{O}$ and IO. Some of them have multi-axis observation capabilities yielding separation of the tropospheric and stratospheric columns. Seven Fourier Transform IR spectrometers (FTIR), also carrying NDSC certification, report the vertical column amount and sometimes the vertical distribution of a bunch of species including $\mathrm{O}_{3}, \mathrm{NO}_{2}, \mathrm{CO}, \mathrm{CH}_{4}, \mathrm{~N}_{2} \mathrm{O}, \mathrm{CO}_{2}$, $\mathrm{HCHO}$, and $\mathrm{H}_{2} \mathrm{O}$. Six microwave (MW) radiometers measure the thermally induced rotational emission of selected species, like $\mathrm{O}_{3}, \mathrm{H}_{2} \mathrm{O}$ and $\mathrm{ClO}$. Ground-based ozone differential absorption lidars (DIAL) and/or electro-chemical ozone sondes yield the vertical distribution of tropospheric and stratospheric ozone, at high and moderate vertical resolution. Aerosol and cloud properties are recorded by lidar and aerosol instruments.

In addition to the instruments operating continuously at ground-based sites, two instruments operated on board the 
Table 2. Ground-based stations contributing to the SCIAMACHY validation effort, and associated SCIAMACHY data products. In the column "core" is indicated when a station is used within the nationally funded core validation programme. The column "netw" identifies stations belonging to the NDSC ("N") and/or Russian/NIS M-124 ("R") networks. "c": column; "p": profile; "ccf": cloud cover fraction; "ctp": cloud top pressure; "AAI": absorbing aerosol index.

\begin{tabular}{|c|c|c|c|c|c|c|c|c|c|c|c|c|c|c|c|c|c|c|c|c|}
\hline station & core & netw & $\begin{array}{l}\text { lat } \\
\left({ }^{\circ} \mathrm{N}\right)\end{array}$ & $\begin{array}{l}\text { long } \\
\left({ }^{\circ} \mathrm{E}\right)\end{array}$ & $\begin{array}{c}\mathrm{O}_{3} \\
(\mathrm{c} / \mathrm{p})\end{array}$ & $\begin{array}{l}\mathrm{NO}_{2} \\
(\mathrm{c} / \mathrm{p})\end{array}$ & $\begin{array}{l}\mathrm{BrO} \\
\text { (c) }\end{array}$ & $\begin{array}{l}\mathrm{OClO} \\
\text { (c) }\end{array}$ & $\begin{array}{l}\mathrm{SO}_{2} \\
\text { (c) }\end{array}$ & $\begin{array}{l}\mathrm{HCHO} \\
\text { (c) }\end{array}$ & $\begin{array}{l}\mathrm{H}_{2} \mathrm{O} \\
(\mathrm{c}, \mathrm{p})\end{array}$ & $\begin{array}{l}\mathrm{CO} \\
\text { (c) }\end{array}$ & $\begin{array}{l}\mathrm{CH}_{4} \\
\text { (c) }\end{array}$ & $\begin{array}{l}\mathrm{N}_{2} \mathrm{O} \\
(\mathrm{c} / \mathrm{p})\end{array}$ & $\begin{array}{l}\mathrm{CO}_{2} \\
\text { (c) }\end{array}$ & $\begin{array}{l}T / p \\
(p)\end{array}$ & UV & $\operatorname{ccf}$ & ctp & AAI \\
\hline Ny-Ålesund & $*$ & $\mathrm{~N}$ & 78.9 & 11.9 & $\mathrm{c}, \mathrm{p}$ & $\mathrm{c}$ & $\mathrm{c}$ & $\mathrm{c}$ & & $\mathrm{c}$ & $\mathrm{c}, \mathrm{p}$ & $\mathrm{c}$ & $\mathrm{c}$ & $\mathrm{c}$ & $\mathrm{c}$ & $\mathrm{p}$ & & & & $\mathrm{x}$ \\
\hline Barentsburg & & $\mathrm{R}$ & 78.1 & 13.2 & $\mathrm{c}$ & & & & & & & & & & & & & & & \\
\hline Thule & $*$ & $\mathrm{~N}$ & 76.5 & -68.8 & $\mathrm{c}, \mathrm{p}$ & $\mathrm{c}$ & & & & & $\mathrm{p}$ & & & & & $\mathrm{p}$ & & & & \\
\hline Summit & $*$ & $\mathrm{~N}$ & 72.2 & -37.8 & $\mathrm{c}, \mathrm{p}$ & $\mathrm{c}$ & $\mathrm{c}$ & $\mathrm{c}$ & & & & & & $\mathrm{p}$ & & & & & & \\
\hline Tiksi & & $\mathrm{R}$ & 71.6 & 128.9 & $\mathrm{c}$ & & & & & & & & & & & & & & & \\
\hline Scoresbysund & $*$ & $\mathrm{~N}$ & 70.5 & -22.0 & $\mathrm{c}, \mathrm{p}$ & $\mathrm{c}$ & & & & & $\mathrm{p}$ & & & & & $\mathrm{p}$ & & & & \\
\hline Andoya/Alomar & $*$ & $\mathrm{~N}$ & 69.3 & 16.0 & $\mathrm{c}, \mathrm{p}$ & $\mathrm{c}$ & $\mathrm{c}$ & $\mathrm{c}$ & & & & & & & & $\mathrm{p}$ & & & $\mathrm{x}$ & $\mathrm{x}$ \\
\hline Murmansk & & $\mathrm{R}$ & 69.0 & 33.1 & $\mathrm{c}$ & & & & & & & & & & & & & & & \\
\hline Kiruna & $*$ & $\mathrm{~N}$ & 67.8 & 20.4 & $\mathrm{c}, \mathrm{p}$ & $\mathrm{c}$ & $\mathrm{c}$ & $\mathrm{c}$ & & $\mathrm{c}$ & $\mathrm{p}$ & & $\mathrm{c}$ & $\mathrm{c}, \mathrm{p}$ & & $\mathrm{p}$ & & & & $\mathrm{x}$ \\
\hline Igarka & & $\mathrm{R}$ & 67.5 & 86.6 & $\mathrm{c}$ & & & & & & & & & & & & & & & \\
\hline Sodankyla & $*$ & $\mathrm{~N}$ & 67.4 & 26.7 & $\mathrm{c}, \mathrm{p}$ & $\mathrm{c}$ & & & & & $\mathrm{p}$ & & & & & $\mathrm{p}$ & & & & \\
\hline SondreStromfjord & $*$ & $\mathrm{~N}$ & 67.0 & -50.7 & $\mathrm{c}$ & & & & & & & & & & & & & & & \\
\hline Zhigansk & $*$ & $\mathrm{~N}$ & 67.2 & 123.4 & $\mathrm{c}$ & $\mathrm{c}$ & & & & & & & & & & & & & & \\
\hline Salekhard & $*$ & $\mathrm{~N}$ & 66.7 & 66.7 & $\mathrm{c}$ & $\mathrm{c}$ & & & & & & & & & & & & & & \\
\hline Pechora & & $\mathrm{R}$ & 65.1 & 57.1 & $\mathrm{c}$ & & & & & & & & & & & & & & & \\
\hline Markovo & & $\mathrm{R}$ & 64.7 & 170.4 & $\mathrm{c}$ & & & & & & & & & & & & & & & \\
\hline Arhangelsk & & $\mathrm{N}, \mathrm{R}$ & 64.6 & 40.5 & $\mathrm{c}$ & & & & & & & & & & & & & & & \\
\hline Vindeln & & & 64.2 & 19.8 & $\mathrm{c}$ & & & & & & & & & & & & & & & \\
\hline Reykjavik & & & 64.0 & -22.6 & $\mathrm{c}$ & & & & & & & & & & & & & & & \\
\hline Orlandet & & & 63.0 & 9.0 & $\mathrm{p}$ & & & & & & $\mathrm{p}$ & & & & & $\mathrm{p}$ & & & & \\
\hline Yakutsk & $*$ & $\mathrm{~N}, \mathrm{R}$ & 62.0 & 129.6 & $\mathrm{c}, \mathrm{p}$ & & & & $\mathrm{c}$ & & $\mathrm{p}$ & & & & & $\mathrm{p}$ & & & & \\
\hline Jokioinen & $*$ & & 60.8 & 23.5 & $\mathrm{c}, \mathrm{p}$ & & & & & & $\mathrm{p}$ & & & & & $\mathrm{p}$ & & & & \\
\hline Harestua & $*$ & $\mathrm{~N}$ & 60.2 & 10.8 & $\mathrm{c}$ & $\mathrm{c}, \mathrm{p}$ & $\mathrm{c}$ & $\mathrm{c}$ & & & & $\mathrm{c}$ & $\mathrm{c}$ & $\mathrm{c}$ & & & & & & \\
\hline Gardermoen & & & 60.1 & 11.0 & $\mathrm{p}$ & & & & & & $\mathrm{p}$ & & & & & $\mathrm{p}$ & & & & \\
\hline Lerwick & & $\mathrm{N}$ & 60.1 & -1.2 & $\mathrm{c}, \mathrm{p}$ & & & & & & $\mathrm{p}$ & & & & & $\mathrm{p}$ & & & & \\
\hline Saint Petersburg & & $\mathrm{R}$ & 60.0 & 30.3 & $\mathrm{c}$ & $\mathrm{c}$ & & & & & & $\mathrm{c}$ & $\mathrm{c}$ & & & & & & & \\
\hline Oslo & & $\mathrm{N}$ & 59.9 & 10.8 & $\mathrm{c}$ & & & & & & & & & & & & & & & \\
\hline Magadan & & $\mathrm{R}$ & 59.6 & 150.8 & $\mathrm{c}$ & & & & & & & & & & & & & & & \\
\hline Vitim & & $\mathrm{R}$ & 59.4 & 112.6 & $\mathrm{c}$ & & & & & & & & & & & & & & & \\
\hline Norrkoping & & & 58.6 & 16.1 & $\mathrm{c}$ & & & & & & & & & & & & & & & \\
\hline Ekaterinburg & & $\mathrm{R}$ & 56.8 & 60.6 & $\mathrm{c}$ & & & & & & & & & & & & & & & \\
\hline Krasnoyarsk & & $\mathrm{R}$ & 56.0 & 92.9 & $\mathrm{c}$ & & & & & & & & & & & & & & & \\
\hline Moscow & & $\mathrm{R}$ & 55.8 & 37.6 & $\mathrm{c}, \mathrm{p}$ & & & & & & $\mathrm{p}$ & & & & & $\mathrm{p}$ & & & & \\
\hline Zvenigorod & & $\mathrm{N}$ & 55.7 & 36.8 & & $\mathrm{c}$ & & & & & $\mathrm{c}$ & $\mathrm{c}$ & $\mathrm{c}$ & & & & & & & \\
\hline Obninsk & & $\mathrm{R}$ & 55.2 & 36.2 & $\mathrm{c}$ & & & & & & & $\mathrm{c}$ & $\mathrm{c}$ & & & & & & & \\
\hline Omsk & & $\mathrm{R}$ & 54.9 & 73.4 & $\mathrm{c}$ & & & & & & & & & & & & & & & \\
\hline Samara & & $\mathrm{R}$ & 53.2 & 50.4 & $\mathrm{c}$ & & & & & & & & & & & & & & & \\
\hline Bremen & $*$ & $\mathrm{~N}$ & 53.1 & 8.9 & $\mathrm{c}$ & $\mathrm{c}$ & $\mathrm{c}$ & $\mathrm{c}$ & & $\mathrm{c}$ & $\mathrm{c}, \mathrm{p}$ & $\mathrm{c}$ & $\mathrm{c}$ & $\mathrm{c}$ & $\mathrm{c}$ & $\mathrm{p}$ & & & & \\
\hline Nikolaevsk & & $\mathrm{R}$ & 53.1 & 140.7 & $\mathrm{c}$ & & & & & & & & & & & & & & & \\
\hline Petropavlovsk & & $\mathrm{R}$ & 53.0 & 158.8 & $\mathrm{c}$ & & & & & & & & & & & & & & & \\
\hline Aberystwyth & $*$ & $\mathrm{~N}$ & 52.7 & -4.1 & $\mathrm{c}$ & $\mathrm{c}$ & & & $\mathrm{c}$ & & & & & & & & & $\mathrm{x}$ & & \\
\hline Irkutsk & & $\mathrm{R}$ & 52.3 & 104.4 & $\mathrm{c}$ & & & & & & & & & & & & & & & \\
\hline Legionowo & & & 52.2 & 20.6 & $\mathrm{p}$ & & & & & & $\mathrm{p}$ & & & & & $\mathrm{p}$ & & & & \\
\hline Lindenberg & & & 52.2 & 14.1 & $\mathrm{c}, \mathrm{p}$ & & & & & & $\mathrm{p}$ & & & & & $\mathrm{p}$ & & & & \\
\hline De Bilt & $*$ & $\mathrm{~N}$ & 52.1 & 5.2 & $\mathrm{c}, \mathrm{p}$ & & & & c & & $\mathrm{p}$ & & & & & $\mathrm{p}$ & $\mathrm{x}$ & & & \\
\hline Bilthoven & & & 52.1 & 5.1 & $\mathrm{p}$ & & & & & & & & & & & & & & & $\mathrm{x}$ \\
\hline Voronezh & & $\mathrm{R}$ & 51.7 & 39.2 & $\mathrm{c}$ & & & & & & & & & & & & & & & \\
\hline Cahirciveen Valentia & & & 51.6 & -10.1 & $\mathrm{c}, \mathrm{p}$ & & & & & & $\mathrm{p}$ & & & & & $\mathrm{p}$ & & & & \\
\hline Uccle & $*$ & $\mathrm{~N}$ & 50.8 & 4.4 & $\mathrm{c}, \mathrm{p}$ & & & & $\mathrm{c}$ & & $\mathrm{p}$ & & & & & $\mathrm{p}$ & & & & \\
\hline Hradec Králové & & $\mathrm{N}$ & 50.2 & 15.8 & $\mathrm{c}$ & & & & & & & & & & & & & & & \\
\hline Camborne & & $\mathrm{N}$ & 50.1 & -5.2 & $\mathrm{c}$ & & & & & & & & & & & & & & & \\
\hline Praha & & & 50.0 & 14.3 & $\mathrm{p}$ & & & & & & $\mathrm{p}$ & & & & & $\mathrm{p}$ & & & & \\
\hline Karaganda & & $\mathrm{R}$ & 49.8 & 73.1 & $\mathrm{c}$ & & & & & & & & & & & & & & & \\
\hline Hohenpeissenberg & $*$ & $\mathrm{~N}$ & 47.8 & 11.0 & $\mathrm{c}, \mathrm{p}$ & & & & $\mathrm{c}$ & & $\mathrm{p}$ & & & & & $\mathrm{p}$ & & & & \\
\hline Zugspitze & $*$ & $\mathrm{~N}$ & 47.4 & 11.0 & $\mathrm{c}, \mathrm{p}$ & $\mathrm{c}$ & $\mathrm{c}$ & & & $\mathrm{c}$ & & & $\mathrm{c}$ & $\mathrm{c}$ & $\mathrm{c}$ & & & & & \\
\hline Bern & & $\mathrm{N}$ & 47.0 & 7.5 & $\mathrm{p}$ & & & & & & $\mathrm{p}$ & & & & & $\mathrm{p}$ & & & & $\mathrm{x}$ \\
\hline Yuzhno Sahalinsk & & $\mathrm{R}$ & 46.9 & 142.7 & $\mathrm{c}$ & & & & & & & & & & & & & & & \\
\hline Arosa & $*$ & $\mathrm{~N}$ & 46.8 & 9.7 & $\mathrm{c}$ & & & & $\mathrm{c}$ & & & & & & & & & & & \\
\hline Jungfraujoch & $*$ & $\mathrm{~N}$ & 46.6 & 8.0 & $\mathrm{c}, \mathrm{p}$ & $\mathrm{c}$ & & & & $\mathrm{c}$ & $\mathrm{c}$ & $\mathrm{c}$ & $\mathrm{c}$ & $\mathrm{c}$ & $\mathrm{c}$ & & & & & $\mathrm{x}$ \\
\hline Payerne & $*$ & $\mathrm{~N}$ & 46.8 & 7.0 & $\mathrm{c}, \mathrm{p}$ & & & & & & $\mathrm{p}$ & & & & & $\mathrm{p}$ & & & & \\
\hline Bordeaux & $*$ & $\mathrm{~N}$ & 44.8 & -0.5 & $\mathrm{c}, \mathrm{p}$ & & & & & & $\mathrm{p}$ & & & & & $\mathrm{p}$ & & & & \\
\hline Bucharest & & & 44.3 & 26.1 & $\mathrm{c}$ & & & & & & & & & & & & & & & \\
\hline Egbert & & & 44.2 & -79.8 & $\mathrm{c}$ & & & & & & & $\mathrm{c}$ & $\mathrm{c}$ & $\mathrm{c}$ & $\mathrm{c}$ & & & & & \\
\hline Monte Cimone & & & 44.2 & 10.7 & & $\mathrm{c}$ & & & & & & & & & & & & & & \\
\hline Haute Provence & $*$ & $\mathrm{~N}$ & 43.9 & 5.7 & $\mathrm{c}, \mathrm{p}$ & $\mathrm{c}$ & $\mathrm{c}$ & & & $\mathrm{c}$ & $\mathrm{p}$ & & & & & $\mathrm{p}$ & & & & \\
\hline Toronto & & $\mathrm{N}$ & 43.7 & -79.4 & $\mathrm{p}$ & & & & & & & & & & & & & & & \\
\hline
\end{tabular}


Table 2. Continued.

\begin{tabular}{|c|c|c|c|c|c|c|c|c|c|c|c|c|c|c|c|c|c|c|c|}
\hline station & core & netw & $\begin{array}{r}\text { lat } \\
\left({ }^{\circ} \mathrm{N}\right)\end{array}$ & $\begin{array}{l}\text { long } \\
\left({ }^{\circ} \mathrm{E}\right)\end{array}$ & $\begin{array}{c}\mathrm{O}_{3} \\
(\mathrm{c} / \mathrm{p})\end{array}$ & $\begin{array}{l}\mathrm{NO}_{2} \\
(\mathrm{c} / \mathrm{p})\end{array}$ & $\begin{array}{l}\mathrm{BrO} \\
\text { (c) }\end{array}$ & $\begin{array}{c}\mathrm{OClO} \\
\text { (c) }\end{array}$ & $\begin{array}{c}\mathrm{SO}_{2} \\
\text { (c) }\end{array}$ & $\begin{array}{l}\mathrm{HCHO} \\
\text { (c) }\end{array}$ & $\begin{array}{l}\mathrm{H}_{2} \mathrm{O} \\
(\mathrm{c}, \mathrm{p})\end{array}$ & $\begin{array}{l}\mathrm{CO} \\
\text { (c) }\end{array}$ & $\begin{array}{l}\mathrm{CH}_{4} \\
\text { (c) }\end{array}$ & $\begin{array}{l}\mathrm{N}_{2} \mathrm{O} \\
(\mathrm{c} / \mathrm{p})\end{array}$ & $\begin{array}{c}\mathrm{CO}_{2} \\
\text { (c) }\end{array}$ & $\begin{array}{l}\mathrm{T} / \mathrm{p} \\
(\mathrm{p})\end{array}$ & UV cef & ctp & AAI \\
\hline Kislovodsk & & $\mathrm{N}$ & 43.7 & 42.7 & $\mathrm{c}$ & $\mathrm{c}$ & & & & & & & & & & & & & \\
\hline Perugia & & & 43.1 & 12.2 & & & & & & & $\mathrm{c}$ & & & & & & & & \\
\hline Vladivostok & & $\mathrm{R}$ & 43.1 & 131.9 & $\mathrm{c}$ & & & & & & & & & & & & & & \\
\hline Issyk Kul & & $\mathrm{N}, \mathrm{R}$ & 42.6 & 77.0 & $\mathrm{c}$ & $\mathrm{c}$ & & & & & $\mathrm{c}$ & & & & & & & & \\
\hline L'Aquila & & & 42.2 & 13.2 & $\mathrm{p}$ & & & & & & $\mathrm{c}, \mathrm{p}$ & & & & & $\mathrm{p}$ & & $\mathrm{x}$ & $\mathrm{x}$ \\
\hline Stara Zagora & & & 42.0 & 25.0 & & $\mathrm{c}$ & & & & & & & & & & & & & \\
\hline Roma & & & 41.0 & 12.0 & $\mathrm{p}$ & & & & & & $\mathrm{c}, \mathrm{p}$ & & & & & & & & $\mathrm{x}$ \\
\hline Thessaloniki & & & 40.4 & 22.6 & $\mathrm{c}$ & & & & & & & & & & & & & & \\
\hline Greenbelt & & $\mathrm{N}$ & 38.9 & -76.7 & $\mathrm{c}$ & & & & & & & & & & & & & & \\
\hline Wallops Island & & $\mathrm{N}$ & 37.9 & -75.5 & $\mathrm{c}$ & & & & & & & & & & & & & & \\
\hline Athens & & & 37.6 & 23.4 & $\mathrm{c}, \mathrm{p}$ & & & & & & $\mathrm{p}$ & & & & & $\mathrm{p}$ & & & \\
\hline Table Mountain & & $\mathrm{N}$ & 34.4 & -117.7 & $\mathrm{p}$ & & & & & & & & & & & & & & \\
\hline Kitt Peak & $*$ & $\mathrm{~N}$ & 31.9 & -111.6 & $\mathrm{c}$ & & & & & & & $\mathrm{c}$ & $\mathrm{c}$ & $\mathrm{c}$ & & & & & \\
\hline Izaña & $*$ & $\mathrm{~N}$ & 28.3 & -16.5 & $\mathrm{c}, \mathrm{p}$ & $\mathrm{c}$ & $\mathrm{c}$ & & & & $\mathrm{c}$ & & & & & & & & \\
\hline Mauna Loa & $*$ & $\mathrm{~N}$ & 19.5 & -155.6 & $\mathrm{c}, \mathrm{p}$ & $\mathrm{c}$ & & & & & & $\mathrm{c}$ & $\mathrm{c}$ & $\mathrm{c}$ & & & & & \\
\hline Mérida & $*$ & $\mathrm{~N}$ & 8.0 & -71.0 & $\mathrm{c}, \mathrm{p}$ & $\mathrm{c}$ & $\mathrm{c}$ & & & & & & & & & $\mathrm{p}$ & & & \\
\hline Paramaribo & $*$ & $\mathrm{~N}$ & 5.8 & -55.2 & $\mathrm{c}, \mathrm{p}$ & $\mathrm{c}$ & $\mathrm{c}$ & & $\mathrm{c}$ & & $\mathrm{p}$ & & & & & $\mathrm{p}$ & $\mathrm{x}$ & & \\
\hline Nairobi & $*$ & $\mathrm{~N}$ & -1.2 & 36.8 & $\mathrm{c}$ & $\mathrm{c}$ & $\mathrm{c}$ & & & & & & & & & & & & \\
\hline Reunion & $*$ & $\mathrm{~N}$ & -21.8 & 55.5 & $\mathrm{c}, \mathrm{p}$ & $\mathrm{c}$ & $\mathrm{c}$ & & & & & $\mathrm{c}$ & $\mathrm{c}$ & $\mathrm{c}$ & & & & & \\
\hline Bauru & $*$ & $\mathrm{~N}$ & -22.3 & -49.0 & $\mathrm{c}$ & $\mathrm{c}$ & & & & & & & & & & & & & \\
\hline Wollongong & $*$ & $\mathrm{~N}$ & -34.4 & 150.9 & $\mathrm{c}$ & $\mathrm{c}$ & & & & & & $\mathrm{c}$ & $\mathrm{c}$ & $\mathrm{c}$ & & & & & \\
\hline Broadmeadows & & & -37.4 & 144.5 & $\mathrm{p}$ & & & & & & $\mathrm{p}$ & & & & & $\mathrm{p}$ & & & \\
\hline Lauder & $*$ & $\mathrm{~N}$ & -45.0 & 169.7 & $\mathrm{c}, \mathrm{p}$ & $\mathrm{c}$ & $\mathrm{c}$ & & & & $\mathrm{p}$ & $\mathrm{c}$ & $\mathrm{c}$ & $\mathrm{c}, \mathrm{p}$ & & $\mathrm{p}$ & $\mathrm{x}$ & & $\mathrm{x}$ \\
\hline Kerguelen & $*$ & $\mathrm{~N}$ & -49.4 & 70.3 & $\mathrm{c}$ & $\mathrm{c}$ & & & & & & & & & & & & & \\
\hline Macquarie & $*$ & $\mathrm{~N}$ & -54.5 & 159.0 & $\mathrm{c}$ & $\mathrm{c}$ & $\mathrm{c}$ & & & & & & & & & & & & \\
\hline Marambia & & $\mathrm{N}$ & -61.0 & -35.6 & $\mathrm{c}, \mathrm{p}$ & $\mathrm{c}$ & $\mathrm{c}$ & $\mathrm{c}$ & & & $\mathrm{p}$ & & & & & $\mathrm{p}$ & & & \\
\hline Vernadsky & $*$ & $\mathrm{~N}$ & -65.2 & -64.3 & $\mathrm{c}$ & & & & & & & & & & & & & & \\
\hline Dumont d'Urville & $*$ & $\mathrm{~N}$ & -66.7 & 140.0 & $\mathrm{c}, \mathrm{p}$ & $\mathrm{c}$ & & & & & $\mathrm{p}$ & & & & & $\mathrm{p}$ & & & \\
\hline Rothera & $*$ & $\mathrm{~N}$ & -67.5 & -68.1 & $\mathrm{c}$ & $\mathrm{c}$ & & & & & & & & & & & & & \\
\hline Neumayer & $*$ & $\mathrm{~N}$ & -70.6 & 8.4 & $\mathrm{c}$ & $\mathrm{c}$ & $\mathrm{c}$ & $\mathrm{c}$ & & & & & & & & & & & \\
\hline Halley & $*$ & $\mathrm{~N}$ & -75.6 & -26.8 & $\mathrm{c}$ & & & & & & & & & & & & & & \\
\hline Arrival Heights & $*$ & $\mathrm{~N}$ & -77.8 & 166.7 & $\mathrm{c}, \mathrm{p}$ & $\mathrm{c}$ & $\mathrm{c}$ & $\mathrm{c}$ & & & $\mathrm{p}$ & $\mathrm{c}$ & $\mathrm{c}$ & $\mathrm{c}, \mathrm{p}$ & & $\mathrm{p}$ & & & \\
\hline Belgrano & & & -77.9 & -34.6 & $\mathrm{c}, \mathrm{p}$ & $\mathrm{c}$ & $\mathrm{c}$ & $\mathrm{c}$ & & & $\mathrm{p}$ & & & & & $\mathrm{p}$ & & & \\
\hline South Pole & & $\mathrm{N}$ & -90.0 & 0.0 & $\mathrm{c}, \mathrm{p}$ & & & & & & & & & & & & & & \\
\hline
\end{tabular}

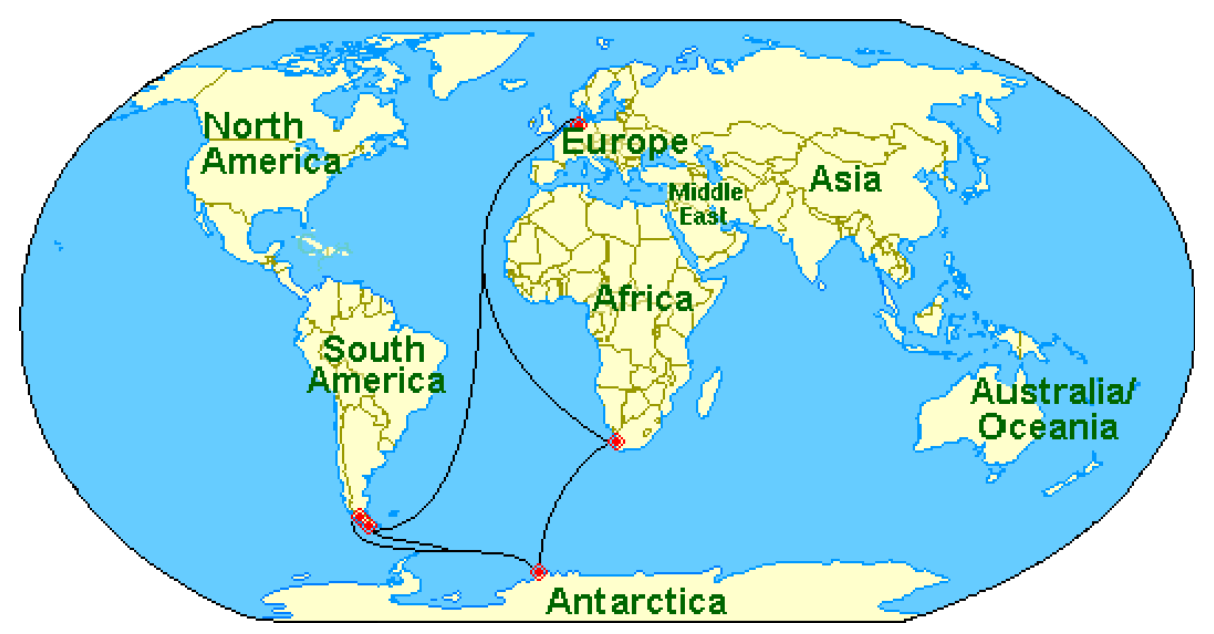

Fig. 4. Route of the first Polarstern cruise (ANT XIX) between November 2001 and June 2002.

German research vessel Polarstern (Fig. 3) to facilitate the validation of SCIAMACHY measurements in remote marine regions (Fig. 4): a MAX-DOAS (Multi-Axis Differential Optical Absorption Spectroscopy) and an FTIR (Fourier Transform InfraRed) instrument. The Polarstern made three cruises within this time period: the first between November 2001 and May 2002, the second between October 2002 and February 2003, the third between October 2003 and July 2004. The moveable MAX-DOAS experiment measured constantly during all three cruises and the investigation of large scale latitudinal cross-sections of atmospheric trace gases were done. The FTIR instrument was operating during the second and third campaign from Bremerhaven to Africa. 

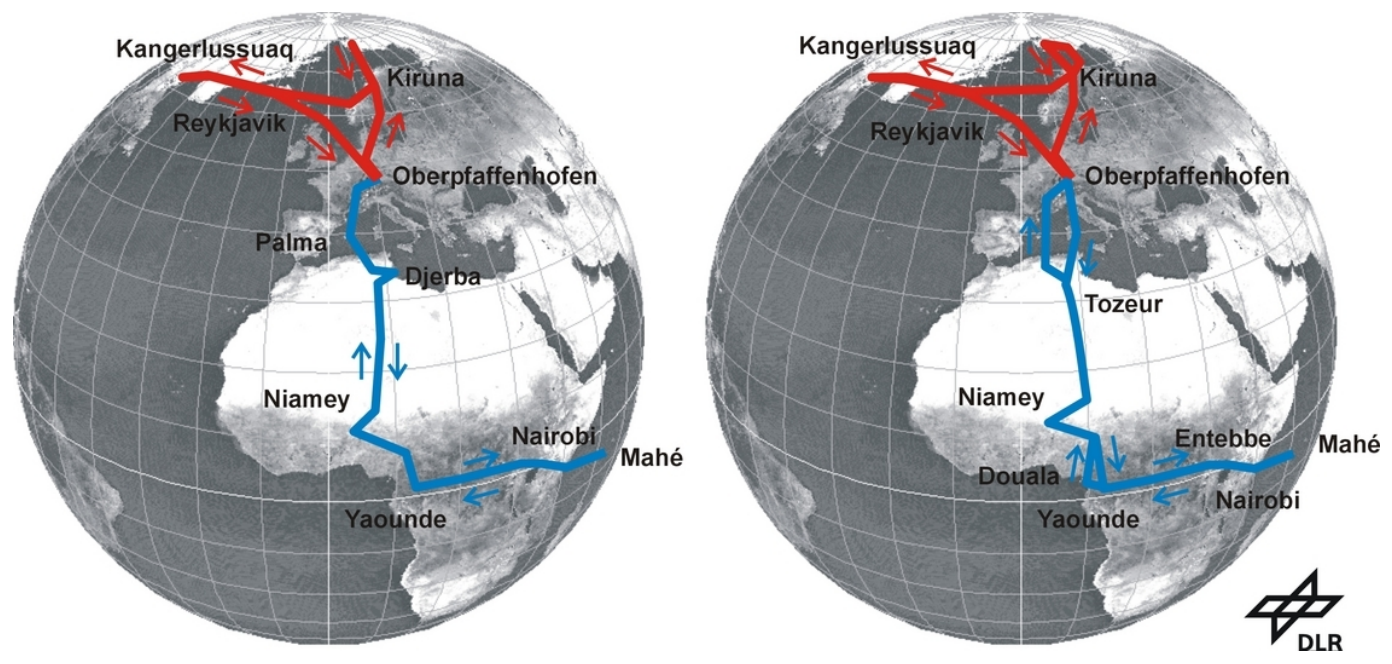

Fig. 5. Flight tracks of the research aircraft Falcon at the SCIA-VALUE campaigns. Left panel, northern track (red): 3-8 September 2002 and southern track (blue) 15-28 September 2002. Right panel, northern track (red) 19 February-3 March, 2003 and southern track (blue) 10-19 March 2003 (image from Fix et al., 2005).

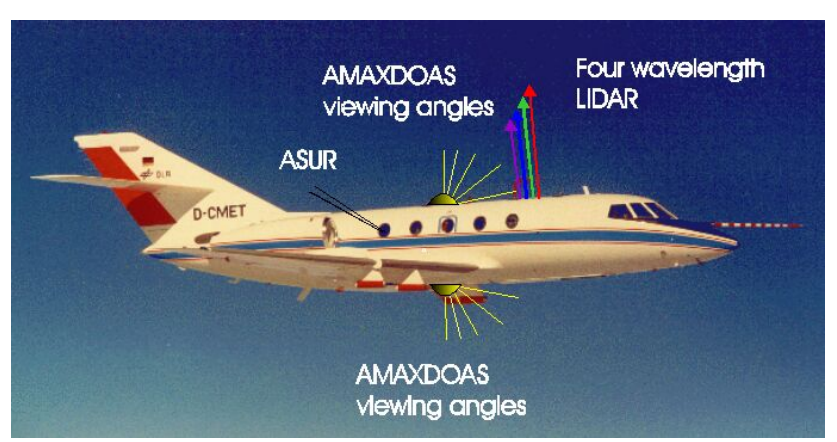

Fig. 6. The Falcon aircraft with the viewing directions of the validation instruments.

\subsection{Airborne campaigns}

The German aircraft validation activities were concentrated on missions with the meteorological research aircraft Falcon 20 (D-CMET) operated by the German Aerospace Center (DLR). Many features make the Falcon an excellent aircraft for the validation experiment. Three large optical windows, two in the bottom and one in the roof enable operation of large lidar experiments both for tropospheric and stratospheric research. Specially manufactured polyethylene windows allow remote sensing in the microwave spectral region. The aircraft carries a data acquisition system and an extensive instrument package capable of measuring position, altitude, static pressure and temperature.

Within the SCIA-VALUE (SCIAmachy VALidation and Utilization Experiment) project two major campaigns with 28 flights were flown in September 2002 and February/March 2003, see Fig. 5. Both campaigns consisted of large-scale latitudinal cross-sections from the polar regions to the tropics as well as longitudinal cross-sections at polar latitudes. To validate SCIAMACHY, three different types of remote sensing instruments were installed on board the Falcon 20 (Fig. 6). The AMAXDOAS (Airborne Multi-Axis Differential Optical Absorption Spectrometer), which is an experiment developed jointly by the Universities of Heidelberg and Bremen, is able to measure tropospheric and stratospheric columns of key gases like $\mathrm{O}_{3}, \mathrm{NO}_{2}, \mathrm{BrO}$ and $\mathrm{OClO}$ absorbing in the UV-visible wavelength range. ASUR (Airborne SUbmillimeter Radiometer) operated by the University of Bremen is a passive microwave sensor. A broad range of molecular lines can be detected containing the molecules that play an important role in the catalytic destruction of ozone. The frequency band includes emission lines of $\mathrm{O}_{3}$, $\mathrm{ClO}, \mathrm{HCl}, \mathrm{HNO}_{3}, \mathrm{~N}_{2} \mathrm{O}, \mathrm{H}_{2} \mathrm{O}, \mathrm{HO}_{2}, \mathrm{CH}_{3} \mathrm{Cl}, \mathrm{NO}, \mathrm{HCN}$, and BrO. The Ozone Lidar Experiment (OLEX) developed and operated by DLR complete the scientific payload of the Falcon. In the zenith looking mode this instrument provides high resolution two-dimensional cross-sections of ozone number densities, aerosol extinction, and cirrus cloud cover information from about $2 \mathrm{~km}$ above aircraft flight level up to a height of $30 \mathrm{~km}$ (Fix et al., 2005).

The stratospheric research aircraft M55-Geophysica is also involved in Envisat validation. It performed two midlatitude campaigns in July and October 2002 from a basis in Forli, Italy and a high latitude campaign in January and March 2003 in Kiruna. For the Envisat validation flights, the M55 was equipped with two sets of instruments. The socalled chemical flights are performed with six in-situ and one remote-sensing instrument, able to measure, among others, concentrations of $\mathrm{H}_{2} \mathrm{O}, \mathrm{O}_{3}, \mathrm{NO}, \mathrm{NO}_{\mathrm{y}}, \mathrm{N}_{2} \mathrm{O}, \mathrm{CH}_{4}, \mathrm{BrO}$, and columns of $\mathrm{O}_{3}$, and $\mathrm{NO}_{2}$ (Kostadinov et al., 2003; Heland 
Table 3. Balloon launches used for SCIAMACHY validation.

\begin{tabular}{|c|c|c|c|}
\hline Payload & Launch dates & Launch site & Target species \\
\hline $\begin{array}{l}\text { MIPAS-B } \\
\text { PI: Fischer }\end{array}$ & $\begin{array}{l}\text { 24 Sep } 2002 \\
7 \text { Dec } 2002 \\
\text { 20 March } 2003 \\
\text { 3 July } 2003\end{array}$ & $\begin{array}{l}\text { Aire sur l'Adour, France } \\
\text { Kiruna, Sweden } \\
\text { Kiruna, Sweden } \\
\text { Kiruna, Sweden }\end{array}$ & $\begin{array}{l}\mathrm{O}_{3}, \mathrm{NO}_{2}, \mathrm{~N}_{2} \mathrm{O}, \mathrm{H}_{2} \mathrm{O}, \mathrm{CO}, \mathrm{CO}_{2} \text {, temperature, } \\
\text { pressure }\end{array}$ \\
\hline $\begin{array}{l}\text { TRIPLE } \\
\text { PI: Fischer }\end{array}$ & $\begin{array}{l}24 \text { Sep } 2002 \\
6 \text { March } 2004 \\
9 \text { June } 2004\end{array}$ & $\begin{array}{l}\text { Aire sur l'Adour, France } \\
\text { Kiruna, Sweden } \\
\text { Kiruna, Sweden }\end{array}$ & $\mathrm{CO}_{2}, \mathrm{CH}_{4}, \mathrm{~N}_{2} \mathrm{O}, \mathrm{NO}_{2}, \mathrm{H}_{2} \mathrm{O}, \mathrm{BrO}$ \\
\hline $\begin{array}{l}\text { LPMA-DOAS } \\
\text { PI: Camy-Peyret }\end{array}$ & $\begin{array}{l}18 \text { Aug } 2002 \\
9 \text { March } 2003 \\
23 \text { March } 2003 \\
9 \text { Oct } 2003 \\
\text { 24 March } 2004\end{array}$ & $\begin{array}{l}\text { Kiruna, Sweden } \\
\text { Kiruna, Sweden } \\
\text { Kiruna, Sweden } \\
\text { Aire sur l'Adour, France } \\
\text { Kiruna, Sweden }\end{array}$ & $\begin{array}{l}\mathrm{O}_{3}, \mathrm{NO}_{2}, \mathrm{OClO}, \mathrm{BrO}, \mathrm{CH}_{4}, \mathrm{~N}_{2} \mathrm{O}, \mathrm{H}_{2} \mathrm{O}, \mathrm{CO} \text {, tem- } \\
\text { perature, pressure, irradiance }\end{array}$ \\
\hline $\begin{array}{l}\text { SAOZ-MIR } \\
\text { PI: Pommereau }\end{array}$ & $\begin{array}{l}23 \text { Feb-4 March } 2003 \\
26 \text { Feb-6 April } 2004\end{array}$ & $\begin{array}{l}\text { Bauru, Brazil } \\
\text { Bauru, Brazil }\end{array}$ & $\mathrm{O}_{3}, \mathrm{NO}_{2}, 2004: \mathrm{H}_{2} \mathrm{O}$ \\
\hline $\begin{array}{l}\text { SAOZ } \\
\text { PI: Goutail }\end{array}$ & $\begin{array}{l}4 \text { Oct } 2002 \\
18 \text { Oct } 2002 \\
9 \text { June } 2004\end{array}$ & $\begin{array}{l}\text { Aire sur l'Adour, France } \\
\text { Aire sur l'Adour, France } \\
\text { Bauru, Brazil }\end{array}$ & $\mathrm{O}_{3}, \mathrm{NO}_{2}$,temperature,pressure \\
\hline $\begin{array}{l}\text { SAOZ + SAOZ-BrO } \\
\text { PI: Goutail/Pirre }\end{array}$ & $\begin{array}{l}\text { 1 Oct } 2002 \\
\text { 23 Feb } 2003 \\
\text { 16 March } 2003 \\
\text { 30 March } 2003 \\
\text { 31 Jan 2004 } \\
\text { 5 Feb 2004 }\end{array}$ & $\begin{array}{l}\text { Aire sur l'Adour, France } \\
\text { Bauru, Brazil } \\
\text { Kiruna, Sweden } \\
\text { Kiruna, Sweden } \\
\text { Bauru, Brazil } \\
\text { Bauru, Brazil }\end{array}$ & $\mathrm{O}_{3}, \mathrm{NO}_{2}$, temperature,pressure, $\mathrm{BrO}$ \\
\hline $\begin{array}{l}\text { FIRS-2 } \\
\text { PI: Chance }\end{array}$ & $\begin{array}{l}20 \text { Oct } 2002 \\
20 \text { Sep } 2003 \\
23 \text { Sep } 2004\end{array}$ & $\begin{array}{l}\text { Ft. Sumner, NM, USA } \\
\text { Ft. Sumner, NM, USA } \\
\text { Ft. Sumner, NM, USA }\end{array}$ & $\begin{array}{l}\mathrm{O}_{3}, \mathrm{H}_{2} \mathrm{O}, \mathrm{N}_{2} \mathrm{O}, \mathrm{NO}_{2}, 2003 / 2004: \mathrm{CO}, \mathrm{CH}_{4}, \mathrm{CO}_{2} \text {, } \\
\text { temperature }\end{array}$ \\
\hline $\begin{array}{l}\text { MANTRA } \\
\text { PI: Strong }\end{array}$ & $\begin{array}{l}3 \text { Sep } 2002 \\
25 \text { Aug } 2004\end{array}$ & $\begin{array}{l}\text { Vanscoy, Canada } \\
\text { Vanscoy, Canada }\end{array}$ & $\begin{array}{l}\mathrm{O}_{3}, \mathrm{NO}_{2}, \mathrm{H}_{2} \mathrm{O}, \mathrm{N}_{2} \mathrm{O}, \mathrm{CH}_{4} \text {, aerosol, pressure, } \\
\text { temperature }\end{array}$ \\
\hline $\begin{array}{l}\text { SALOMON } \\
\text { PI: Renard }\end{array}$ & $\begin{array}{l}19 \text { Sep } 2002 \\
4 \text { March } 2002\end{array}$ & $\begin{array}{l}\text { Aire sur l'Adour, France } \\
\text { Kiruna, Sweden }\end{array}$ & $\mathrm{O}_{3}, \mathrm{NO}_{2}, \mathrm{OClO}$, aerosol \\
\hline $\begin{array}{l}\text { SPIRALE } \\
\text { PI: Pirre }\end{array}$ & $\begin{array}{l}2 \text { Oct } 2002 \\
21 \text { Jan } 2003\end{array}$ & $\begin{array}{l}\text { Aire sur l'Adour, France } \\
\text { Kiruna, Sweden }\end{array}$ & $\mathrm{O}_{3}, \mathrm{NO}_{2}, \mathrm{CO}, \mathrm{CH}_{4}$ \\
\hline $\begin{array}{l}\text { SDLA-LAMA } \\
\text { PI: Pirre }\end{array}$ & 8 Aug 2002 & Kiruna, Sweden & $\mathrm{H}_{2} \mathrm{O}, \mathrm{CH}_{4}$ \\
\hline $\begin{array}{l}\text { ELHYSA } \\
\text { PI: Ovarlez }\end{array}$ & 11 March 2004 & Kiruna, Sweden & $\mathrm{H}_{2} \mathrm{O}, \mathrm{CH}_{4}$ \\
\hline $\begin{array}{l}\text { AMON } \\
\text { PI: Renard }\end{array}$ & 1 March 2003 & Kiruna, Sweden & $\mathrm{O}_{3}, \mathrm{NO}_{2}, \mathrm{OClO}$ \\
\hline $\begin{array}{l}\mu \mathrm{RADIBAL} \\
\text { PI: Brogniez }\end{array}$ & 8 March 2004 & Kiruna, Sweden & aerosol \\
\hline $\begin{array}{l}\text { LPMA-IASI } \\
\text { PI: Renard }\end{array}$ & 6 Aug 2002 & Kiruna, Sweden & $\mathrm{H}_{2} \mathrm{O}(\mathrm{c}), \mathrm{CO}_{2}(\mathrm{c}) \mathrm{CO}(\mathrm{c}), \mathrm{O}_{3}(\mathrm{c}), \mathrm{N}_{2} \mathrm{O}(\mathrm{c}), \mathrm{CH}_{4}(\mathrm{c})$ \\
\hline
\end{tabular}

et al., 2003). For the the so-called cloud/aerosol flights, the remote-sensing instrument was replaced by six instruments for the characterisation of aerosol and cloud properties. Although the in-situ instruments remain on-board, these flights were optimised for measuring the cloud and aerosol properties.

Within the MOZAIC program (Marenco et al., 1998) started in 1994, five long-range Airbus A340 aircrafts are equipped with in situ-instruments measuring $\mathrm{O}_{3}, \mathrm{H}_{2} \mathrm{O}, \mathrm{CO}$ and $\mathrm{NO}_{\mathrm{y}}$. They provide data from all over the world along the flight tracks at the upper troposphere, lower stratosphere altitude level from 9-12 km and down to the ground around 60 airports. These measurements are a unique dataset at the tropopause region and will be useful especially for development and validation of products distinguishing between troposphere and stratosphere. 
Table 4. Satellite instruments used in the core validation for intercomparison with SCIAMACHY.

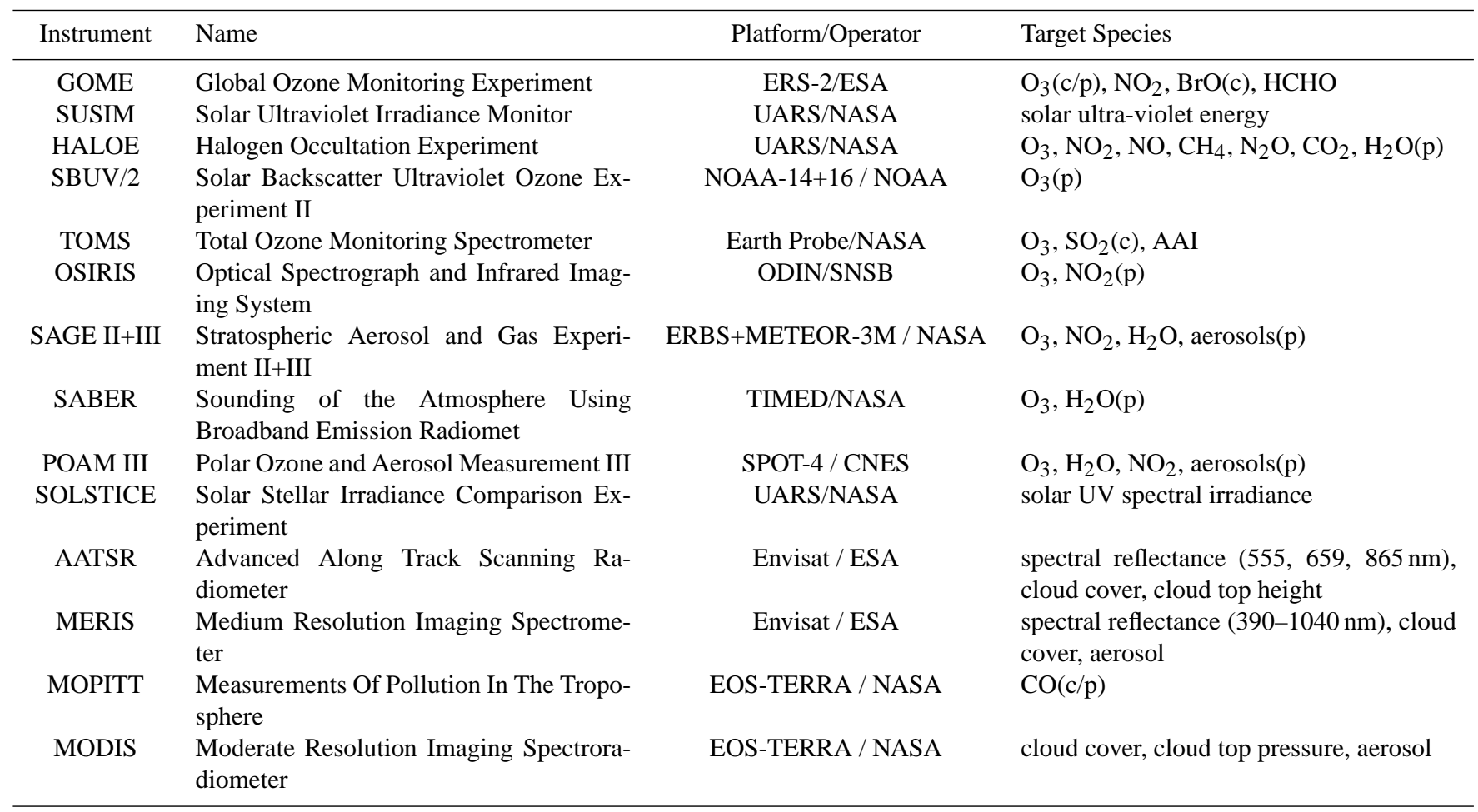

\subsection{Balloon campaigns}

Balloon-borne measurements provide snapshot type vertical profile measurements of very high precision. The dedicated balloon campaigns for the atmospheric chemistry instruments MIPAS, GOMOS and SCIAMACHY are financed by ESA, DLR and the French space agency CNES (Centre National d' Etudes Spatiales), the costs and responsibilities are shared according to an agreement between the three agencies. Part of this agreement is to use all balloon flights as much as possible for all three satellite instruments.

CNES provides the facilities and staff for launching scientific payloads with large stratospheric balloons from dedicated stations. The availability of the CNES equipment is an important constraint for the implementation of campaigns. Within the ACVT sub-group ESABC, the involved scientists from the balloon teams and representatives of the agencies frequently met to organize the Envisat validation balloon campaigns. The launch sites and campaign times are selected to cover mid-latitudes, northern latitudes and the tropics during several seasons as much as possible within the available resources.

Until May 2005, SCIAMACHY validation measurements were performed during 16 balloon campaigns from launch sites in Kiruna, Sweden (August 2002, December 2002/January 2003, March 2003, July 2003, March 2004, June 2004),
Aire sur l'Adour, France (September/October 2002, October 2003), Bauru, Brazil (February/March 2003, January/April 2004, June 2004), Vanscoy, Canada (September 2002, August 2004), and Fort Sumner, New Mexico, USA (October 2002, September 2003, September 2004). Table 3 lists the payloads and the measured species relevant for SCIAMACHY.

Further ESABC supported campaigns are already planned in Brazil (June/July 2005), and Kiruna (January/February 2006). With these campaigns, high latitudes in summer with usual conditions and in spring with the possibility of ozone depletion are covered as well as mid-latitudes and tropical regions.

Explicitly funded for the validation of SCIAMACHY are the LPMA/DOAS (combining a Limb Profile Monitoring of the Atmosphere FTIR and a Differential Optical Absorption Spectrometry instrument), the TRIPLE (combining a resonance fluorescence $\mathrm{ClO} / \mathrm{BrO}$ instrument, an in-situ Fluorescence Induced Stratospheric Hygrometer FISH, a cryogenic total air sampler (BONBON) and a tunable diode laser measuring $\mathrm{H}_{2} \mathrm{O}$ and $\mathrm{CH}_{4}$ ) and the MIPAS-B (Michelson Interferometer for Passive Atmospheric Sounding - balloon version) balloon gondolas. These constitute the German contribution to the balloon-borne validation of Envisat.

These three balloon payloads together measured atmospheric profiles of $\mathrm{O}_{3}, \mathrm{NO}_{2}, \mathrm{OClO}, \mathrm{BrO}, \mathrm{CH}_{4}, \mathrm{~N}_{2} \mathrm{O}, \mathrm{H}_{2} \mathrm{O}$, 
Table 5. Overview of the software versions ("sv") of the NRT and OL operational processors, the months in which they became operational ("oper"), and the time range of the data processed with this processor version ("range"). Note that not all data within the listed time ranges are processed, as can be seen in Figs. 7 and 8.

\begin{tabular}{|c|c|c|c|c|c|c|c|c|}
\hline \multicolumn{3}{|c|}{ NRT $0-1 b$} & \multicolumn{3}{|c|}{ NRT 1b-2 } & \multicolumn{3}{|c|}{ OL $1 b-2$} \\
\hline sv & oper & range & sv & oper & range & sv & oper & range \\
\hline 3.51 & July 2002 & $2002-07 / 2002-08$ & 3.53 & Aug 2002 & $2002-08 / 2002-12$ & 2.1 & March 2004 & $2002-07 / 2002-11$ \\
\hline 4.00 & Dec 2002 & $2002-12 / 2003-04$ & 4.00 & Dec 2002 & $2002-12 / 2003-04$ & 2.4 & Sep 2004 & 2004-09/2004-11 \\
\hline 4.01 & April 2003 & 2003-04/2003-09 & 4.01 & April 2003 & 2003-04/2003-09 & 2.5 & Dec 2004 & $2004-12 / 2005-03$ \\
\hline $4.02 \mathrm{~b}$ & Dec 2003 & $2002-07 / 2002-08$ & 4.03 & Aug 2003 & $2003-08 / 2004-03$ & & & \\
\hline 4.03 & Aug 2003 & 2003-09/2004-03 & 5.01 & March 2004 & $2002-07 / 2003-08$ & & & \\
\hline 5.00 & Jan 2004 & $2002-07 / 2002-10$ & & & 2004-02/2004-08 & & & \\
\hline 5.01 & March 2004 & $2002-08 / 2002-12$ & 5.04 & Aug 2004 & $2002-07 / 2005-03$ & & & \\
\hline & & 2003-02/2003-04 & & & & & & \\
\hline & & 2004-03/2004-08 & & & & & & \\
\hline 5.04 & Aug 2004 & $2002-07 / 2003-05$ & & & & & & \\
\hline & & 2003-07/2004-05 & & & & & & \\
\hline & & $2004-07 / 2005-03$ & & & & & & \\
\hline
\end{tabular}

$\mathrm{CO}, \mathrm{CO}_{2}$, temperature and pressure which will allow to validate corresponding parameters measured by SCIAMACHY during collocated overpasses of the satellite. By radiometric calibration of the DOAS instruments on board the LPMA/DOAS gondola, solar irradiances and limb radiances are determined for level 1 validation.

\subsection{Satellite intercomparisons}

Measurements of relevant parameters by independent instruments on board other satellite platforms facilitate the required pole-to-pole validation for many of the SCIAMACHY products for all seasons. Several satellite instruments are available for comparison with results from the Envisat atmospheric chemistry experiments. In most cases satellite measurements provide near global coverage and, therefore, are well suited for global validation in space and time.

Table 4 lists the satellite instruments used for the validation of SCIAMACHY products. SCIAMACHY's precursor GOME on board ERS-2 follows Envisat with a thirty minutes delay. Since the GOME channels are almost identical to the UV-visible channels of SCIAMACHY, GOME is the first choice for validating UV-visible nadir products. Unfortunately, since June 2003 the tape recorder on board ERS-2 stopped recording data, so that GOME measurements are restricted to areas where direct downlink of data is possible (currently about $30 \%$ is retrieved). TOMS and SBUV II as nadir looking instruments provide total columns. HALOE, SAGE II/III, and POAM III are solar occultation instruments, providing trace gas profiles at sunset and sunrise. SABER observes infrared emissions in limb, retrieving ozone and water vapour profiles. OSIRIS also operates in limb mode, providing ozone profiles. SUSIM and SOLSTICE results are used for comparison with solar irradiance measurements, needed to check the radiometric calibration of SCIAMACHY.

In addition intercomparisons are performed between the three atmospheric chemistry instruments on board Envisat: MIPAS, GOMOS and SCIAMACHY.

\section{SCIAMACHY data}

\subsection{Operational products}

The raw data of the SCIAMACHY instrument are sent from Envisat to the receiving stations, reformatted there and stored as "level 0" products. Rearranged and combined with the appropriate calibration information, they are converted by the SCIAMACHY data processor to the so-called "level 1b" products. These contain the measured spectra as raw data and the calibration information needed to derive spectra in physical units.

The level $1 \mathrm{~b}$ products are the basis to derive atmospheric parameters from the SCIAMACHY measurements. Two operational processors are implemented to derive these parameters: the Near-Real Time (NRT) and the Offline (OL) level $1 \mathrm{~b}-2$ processor. The NRT processor derives trace gas columns, cloud and aerosol information from SCIAMACHY nadir measurements, the OL processor additionally contains trace gas vertical profiles derived from the limb measurement.

Several software updates to the operational processors have been performed since the launch of Envisat. An overview of the software version numbers and the periods to which they apply is given in Table 5.

The first (re-)processing of the entire mission started in March 2004 with software version 5.01. A later software 


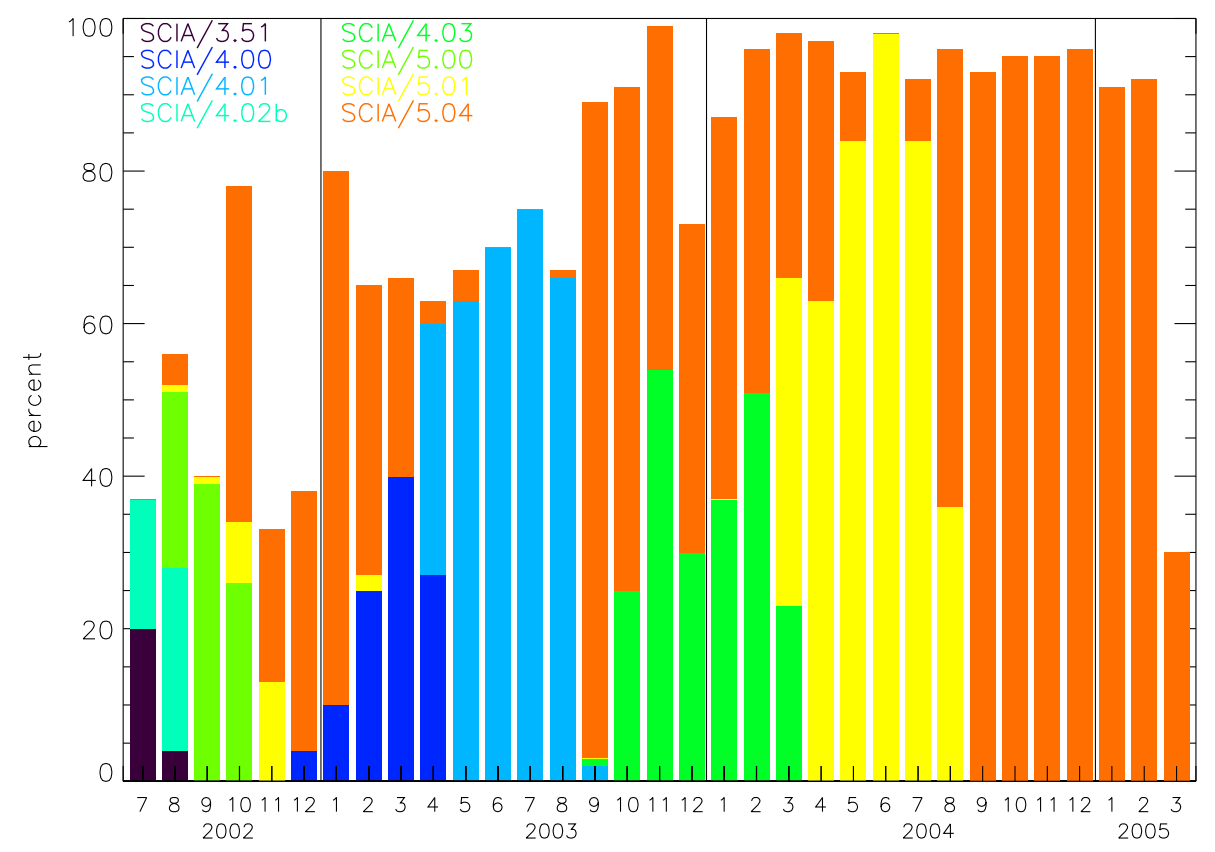

Fig. 7. The fraction of level $1 \mathrm{~b}$ orbits available in March 2005 for several software versions. Orbits, processed with more than one software version, have only been counted for the latest software version. This plot does not account for Envisat or SCIAMACHY off-time, so part of the "missing" data will actually never become available (see Fig. 1). Software versions 5.01 and 5.04 have been used for reprocessing the entire mission.

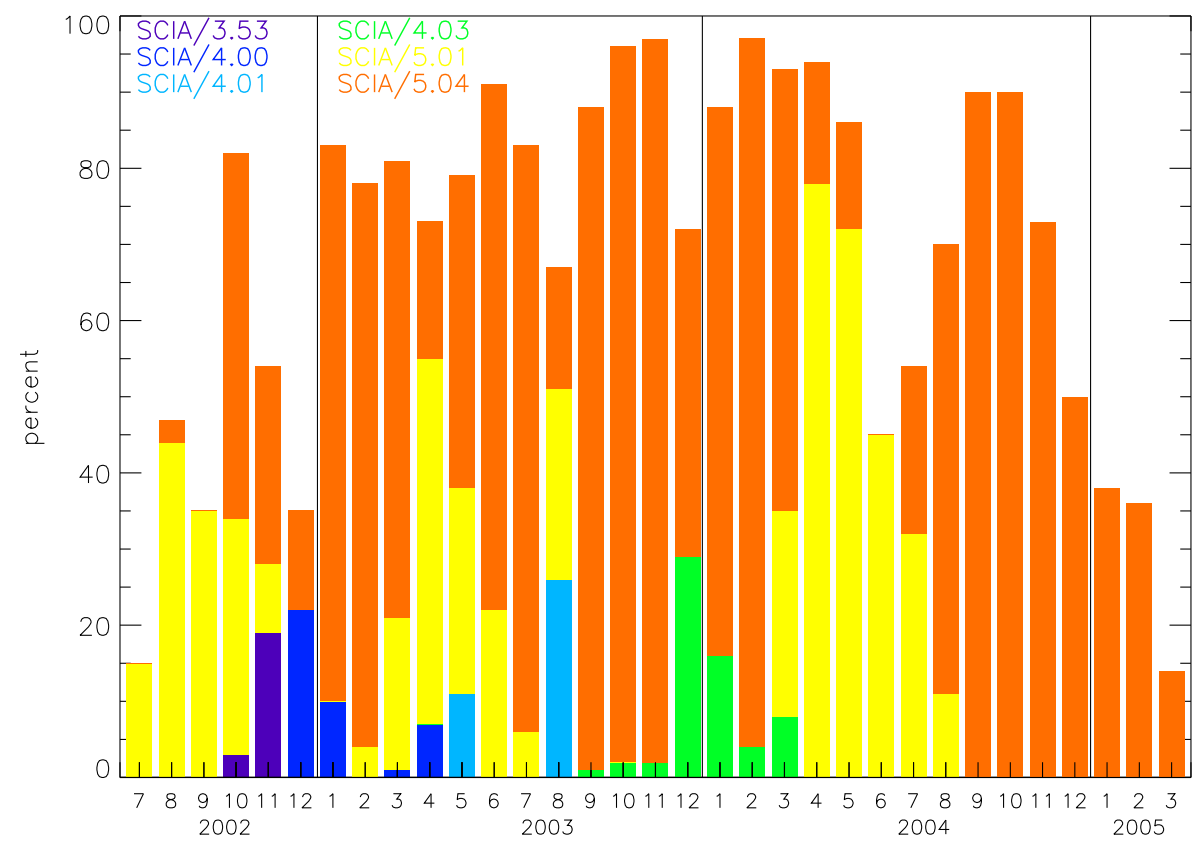

Fig. 8. The same as Fig. 7, but now for level 2 orbits. 
version (5.04) was introduced in August 2004 and the reprocessing was continued with this version. Note that not all data within the listed time ranges are processed. This can be seen in Figs. 7 and 8, which shows the fraction actually available to the validation teams for each processor version. For the latest level 2 NRT version (5.04, orange bars in Fig. 8) this fraction is on average $45 \%$ between July 2002 and March 2005.

The auxiliary files and the initialisation files used in the processors are different for different software versions. Periods with erroneous initialisation or auxiliary files are in principle listed in ESA's data disclaimer. The data disclaimers can be found on ESA's web site http://envisat.esa. int/dataproducts/availability/disclaimers.

SCIAVALIG, with input from the validation community, has defined a validation reference set: a small subset of SCIAMACHY measurements coinciding with selected validation measurements between July 2002 and April 2003. With this reference set it should be possible to derive a first statement of the product quality from comparison with the validation measurements after every processor upgrade, provided that this set is processed first.

For the second workshop of Atmospheric Chemistry Validation of Envisat in May 2004 (ACVE-2), the 2002 part of the reference set was processed to level $1 \mathrm{~b}$ and level 2 NRT and OL products with the most recent processors (NRT: 5.01 and OL: 2.1, see Table 5). The OL products contained only ozone and $\mathrm{NO}_{2}$ profiles. The NRT products contained entries for all planned nadir products, but only $\mathrm{O}_{3}, \mathrm{NO}_{2}, \mathrm{BrO}$, and cloud cover fraction could be used for comparison studies; the retrieval of the other species was suffering from calibration issues or from errors in the processors.

\subsection{Scientific products}

Many institutes interested in the SCIAMACHY project developed their own retrieval algorithms to derive atmospheric products from SCIAMACHY measurements as an alternative for the operational products. These non-operational products, hereafter called "scientific products", are more advanced than the operational ones. For many atmospheric parameters, scientific products are the only ones available at the time of writing of this paper. The availability of the scientific products varies. For some products, almost complete datasets exist. This is the case for $\mathrm{O}_{3}, \mathrm{NO}_{2}, \mathrm{BrO}, \mathrm{OClO}, \mathrm{SO}_{2}$, and $\mathrm{H}_{2} \mathrm{O}$ columns, and for $\mathrm{O}_{3}, \mathrm{NO}_{2}$ and $\mathrm{BrO}$ profiles.

The improvement of scientific products usually goes faster than that of the operational products, because changes can be faster implemented without the restricting needs of an operational processor regarding design, stability and documentation. The knowledge gained by validation and further development of scientific products can help considerably in the improvement of the operational products.

Table 1 gives an overview of the current availability of operational and scientific products. Section 6 gives a short sum- mary of first validation results, including information about the institutes developing the scientific products.

\section{Validation results}

This section contains a summary of validation results reported during the Second Workshop on the Atmospheric Chemistry Validation of Envisat (ACVE-2; May 2004, Frascati), during the SCIAMACHY Validation Workshop (December 2004, Bremen), and in the literature till October 2005. It must be noted that for all products the data sets suitable for evaluation were too limited to cover all geophysical states of interest. Qualitative and quantitative statements hereafter are valid only for the data sets and algorithm versions available at the time of the investigation.

\subsection{Level 1 products}

The solar spectral irradiance measured by SCIAMACHY has been compared with the high-resolution Kurucz solar spectrum (Skupin et al., 2005) and with balloon measurements (Gurlit et al., 2005). These comparisons have revealed deficiencies in the absolute radiometric calibration of the solar irradiance, with offsets ranging between $6 \%$ and $15 \%$. Comparisons of the spectral reflectance, i.e., the ratio of the earth radiance at nadir and the solar irradiance with GOME, MERIS and modelled reflectance spectra, show wavelength dependent offsets varying between $5 \%$ and $25 \%$ (Acarreta and Stammes, 2005; van Soest et al., 2005). Such offsets cannot be explained by absolute calibration errors of the solar irradiance alone, suggesting also uncertainties with the absolute calibration of the earth radiance different from solar irradiance calibration errors. Reflectance comparisons have also revealed the presence of residual polarisation sensitivity structures in the spectra even after application of the polarisation correction (Tilstra and Stammes, 2005).

According to recent studies, the calibration improves greatly by recalculating calibration parameters from onground measurements and extended analysis of in-flight measurements. With these improvements, the mean solar irradiance for SCIAMACHY channels 3-6 is expected to agree with the Kurucz data to within $\pm 2-3 \%$, and for channels 1 and 2 within $\pm 5-10 \%$ (Skupin et al., 2005). The reflectance is expected to agree within $\pm 2-7 \%$ for channels $1-6$. However, these changes are not yet implemented in the operational chain at this time.

The near-infrared channels 7 and 8 are hampered by various effects, the most important being: varying ice layer on the cooled detectors causing transmission loss and further effects; light-leak in channel 7; non-linearity of the detector electronics; variable dark current (Lichtenberg et al., 2005). The current calibration of these channels is insufficient to start the validation of reflectance data in this wavelength region. 
Table 6. Summary of the validation status per product in March 2005 for the latest software versions. Details can be found in Sect. 6, applicable subsections are listed in the second column ("sect"). "B" means average systematic deviation between SCIAMACHY and correlative measurements. If a range is give here, the systematic deviation depends on the geophysical state and/or measurement parameters. If the sign of the bias is negative, SCIAMACHY is on average lower than the correlative measurement. "S" means average scatter of the deviations. Software version numbers (for operational products) and responsible institutes (for scientific products) are shown in parentheses. "BIRA"=BIRA/IASB Brussels, "Heid"=IUP-Heidelberg, "IFE"=IFE/IUP-Bremen, "KNMI"=KNMI De Bilt, "SAO"=SAO Harvard, "SRON"=SRON Utrecht.

\begin{tabular}{|c|c|c|c|}
\hline product & sect & average agreement & special features / remarks \\
\hline $\begin{array}{l}\text { solar irradiance spectrum } \\
\text { (NRT 5.04, SciaL1C 2.2.9) }\end{array}$ & 6.1 & B: $+6 /+13 \%$ & \\
\hline $\begin{array}{l}\text { nadir earth radiance spectrum } \\
\text { (NRT 5.04, SciaL1C 2.2.9) }\end{array}$ & - & $\mathrm{n} / \mathrm{a}$ & no validation sources \\
\hline $\begin{array}{l}\text { limb earth radiance spectrum } \\
\text { (NRT 5.04, SciaL1C 2.2.9) }\end{array}$ & - & $\mathrm{n} / \mathrm{a}$ & no validation sources \\
\hline $\begin{array}{l}\text { spectral reflectance } \\
\text { (NRT 5.04, SciaL1C 2.2.9) }\end{array}$ & 6.1 & B: $-5 /-25 \%$ & residual polarisation structures \\
\hline earth fractional polarisation & - & unknown & \\
\hline $\mathrm{O}_{3}$ column (NRT 5.04) & 6.2 .1 & B: $-2 /-10 \%$ & $\begin{array}{l}\text { dependence on SZA, cloud cover; known errors in re- } \\
\text { trieval for Oct-Dec }\end{array}$ \\
\hline $\mathrm{O}_{3}$ column (OL 2.5) & - & unknown & \\
\hline $\mathrm{O}_{3}$ column (KNMI) & 6.2 .1 & B: $-1 /-1.5 \%, S: 5 \%$ & \\
\hline $\mathrm{NO}_{2}$ column (NRT 5.04) & 6.2 .2 & $\begin{array}{l}\mathrm{B} \& \mathrm{~S}: \sim 10^{14} \mathrm{molec} / \mathrm{cm}^{2} \\
\mathrm{~B}:>+1.5 \cdot 10^{15} \mathrm{molec} / \mathrm{cm}^{2}\end{array}$ & $\begin{array}{l}\text { over clean areas July-Sept. } \\
\text { otherwise; known errors in retrieval for Oct.-Dec. }\end{array}$ \\
\hline $\mathrm{NO}_{2}$ column $(\mathrm{OL} 2.5)$ & - & unknown & \\
\hline $\mathrm{NO}_{2}$ column (BIRA,Heid,IFE,KNMI) & 6.2 .2 & $\begin{array}{l}\text { B: }-7 \cdot 10^{14} / \\
+10^{14} \mathrm{molec} / \mathrm{cm}^{2}\end{array}$ & bias depends on retrieval method and settings \\
\hline BrO slant column (NRT 5.04) & 6.2 .3 & not specified & Large positive bias for small SCDs \\
\hline BrO slant column (OL 2.5) & - & unknown & \\
\hline BrO slant column (BIRA,Heid,IFE,SAO) & 6.2 .3 & not specified & Consistent with GB (2 stations) and GOME \\
\hline $\mathrm{SO}_{2}$ column (BIRA,IFE) & 6.2 .4 & unknown & Qualitatively ok; special events needed for validation \\
\hline OClO slant column (Heid,IFE) & 6.2 .5 & not specified & consistent with GOME \\
\hline HCHO column (IFE,SAO) & - & unknown & \\
\hline $\mathrm{H}_{2} \mathrm{O}$ column (IFE) & 6.2 .6 & $\begin{array}{l}\text { B: }-0.05 \mathrm{~g} / \mathrm{cm}^{2} \\
\text { S: } 0.5 \mathrm{~g} / \mathrm{cm}^{2}\end{array}$ & \\
\hline $\mathrm{N}_{2} \mathrm{O}$ column (IFE) & 6.3 & S: $20 \%$ & \\
\hline CO column (Heid,IFE,SRON) & 6.3 & S: $30 \%$ & \\
\hline $\mathrm{CO}_{2}$ column (IFE) & 6.3 & unknown & validation too limited \\
\hline $\mathrm{CH}_{4}$ column (Heid,IFE,SRON) & 6.3 & S: $5 \%$ & \\
\hline cloud cover (NRT 5.04) & 6.2 .7 & unknown & good, compared to scientific products \\
\hline cloud cover (OL 2.5) & - & unknown & \\
\hline cloud cover (KNMI) & 6.2 .7 & unknown & good, compared to NRT 5.04 \\
\hline cloud top pressure (KNMI) & 6.2 .7 & $\mathrm{~B}: 100 \mathrm{hPa}$ & with respect to MODIS \\
\hline Absorbing Aerosol Index (KNMI) & 6.2 .8 & not specified & compares well with TOMS; sens. to lv1 errors \\
\hline Aerosol Optical Thickness (IFE) & 6.2 .8 & not specified & compares well with MERIS; sens. to lv1 errors \\
\hline \multirow[t]{3}{*}{$\mathrm{O}_{3}$ profile (OL 2.5; limb) } & 6.4 .1 & B: 0 & $>24 \mathrm{~km}$ \\
\hline & & B: $-15 \%$ & $20-24 \mathrm{~km}$ \\
\hline & & S: $13-18 \%$ & $25-40 \mathrm{~km} ; 20 \%$ of profiles show unrealistic features \\
\hline \multirow{2}{*}{$\mathrm{O}_{3}$ profile (IFE $\left.1.61 ; \mathrm{limb}\right)$} & 6.4 .1 & $\mathrm{~B}:-3 /-6 \%$ & 16-40 km; zigzag shaped difference profiles \\
\hline & & S: $10 \%$ & $20-35 \mathrm{~km}$ \\
\hline $\mathrm{O}_{3}$ profile (KNMI; nadir) & - & unknown & depends on future improvement of level 1 data \\
\hline \multirow{3}{*}{$\mathrm{NO}_{2}$ profile (OL 2.5; limb) } & 6.4 .2 & B: $-20 /+5 \%$ & $60 \mathrm{~S}-70 \mathrm{~S}, 19-36 \mathrm{~km}$ \\
\hline & & B: $+10 /+60 \%$ & $25 \mathrm{~N}-30 \mathrm{~N}, 25-36 \mathrm{~km}$ \\
\hline & & B: $-35 /+25 \%$ & $40 \mathrm{~N}-53 \mathrm{~N}, 19-36 \mathrm{~km}$ \\
\hline $\mathrm{NO}_{2}$ profile (IFE,SAO; limb) & 6.4 .2 & $\mathrm{~B}:<15 \%$ & $22-33 \mathrm{~km}$; compares reasonable to balloon profiles \\
\hline BrO profile (IFE,SAO; limb) & 6.4 .3 & unknown & possible problems below $20 \mathrm{~km}$ \\
\hline OClO profile (SAO; limb) & - & unknown & look geophysically consistent \\
\hline $\mathrm{H}_{2} \mathrm{O}$ profile (IFE; limb) & - & unknown & \\
\hline
\end{tabular}




\subsection{Level 2 products from nadir UV-visible}

Apparent slant column amounts of $\mathrm{O}_{3}, \mathrm{NO}_{2}, \mathrm{BrO}, \mathrm{OClO}$, $\mathrm{SO}_{2}, \mathrm{HCHO}$ and $\mathrm{H}_{2} \mathrm{O}$ are retrieved from SCIAMACHY UV-visible spectra measured at nadir. When the vertical distribution of the constituents controlling the optical path through the atmosphere is known a priori, slant columns can be converted into vertical columns using an air mass factor (AMF) calculated with a radiative transfer model. Also retrieved from nadir UV-visible data are the fractional cloud cover, cloud top pressure, absorbing aerosol index (AAI), and aerosol optical thickness (AOT).

\subsection{1 $\mathrm{O}_{3}$ column}

Preliminary validations based on sporadic sets of SCIAMACHY $\mathrm{O}_{3}$ columns processed with versions 5.01 and 5.04 of the NRT processor were overviewed by Lambert et al. (2004a). They concluded that the NRT $\mathrm{O}_{3}$ column products offer in most cases the level of quality that could be expected from this processor based on version 2 of the GOME Data Processor (GDP). For several seasons and latitudes, the agreement to ground-based networks and satellite measurements is indeed within the $2-10 \%$ range, with a global tendency to underestimate correlative data by a few percent. Ground-based comparisons of the SCIAMACHY NRT $\mathrm{O}_{3}$ columns confirm the expected presence of errors inherited from GDP 2, like dependences on the solar zenith angle, season and viewing angle. The apparent absence of meridional structures between SCIAMACHY NRT 5.01/5.04 and ground-based total $\mathrm{O}_{3}$ is surprising at first glance but it can be the result of compensating errors which vary with time and latitude, e.g. errors associated with rotational Raman scattering (RRS) and with the temperature dependence of the $\mathrm{O}_{3}$ absorption cross-section. Between October and December, unexpectedly, much larger errors appear than those reported for GOME. Those errors correlate with the cloud fraction, ghost vertical column and air mass factor, and they show up at several latitudes. This explains apparent differences between individual comparison results and vindicates the maintenance of a network-based effort instead of only a few "representative" stations. It is expected that the OL SCIAMACHY processor will be upgraded in 2005 to a GDP 4-like version, including a better treatment of RRS, of the cross-sections temperature dependence and of the air mass factor, and therefore significant improvements are anticipated. Comparisons with GOME retrievals from IFE/IUP-Bremen (WF-DOAS 1.0) confirm these results and additionally show that the dependencies of the deviations on solar zenith angle, latitude, and total ozone disappear when SCIAMACHY total ozone is retrieved with an algorithm equivalent to GOME WF-DOAS 1.0 (Bracher et al., 2005a).

SCIAMACHY $\mathrm{O}_{3}$ column data sets have also been produced by scientific processors developed at BIRA-IASB, IUP/Bremen and KNMI. Those processors are based on re- cent $\mathrm{GOME} \mathrm{O}_{3}$ column retrieval algorithms (GDOAS, Spurr et al., 2004; TOGOMI, Valks and van Oss, 2003; WF-DOAS, Coldewey-Egbers et al., 2005) which have demonstrated to bring the SZA/season/latitude dependencies of the GOME $\mathrm{O}_{3}$ column product down to the " $1 \%$ level", that is, to the level of accuracy that can be reached with well-maintained and calibrated ground-based sensors. Those algorithms have also proven to be stable and relatively insensitive to instrumental degradation over the entire GOME lifetime, enabling accurate $\mathrm{O}_{3}$ trend monitoring. First validation of preliminary SCIAMACHY $\mathrm{O}_{3}$ columns generated by the scientific algorithm developed at KNMI shows a good agreement of 1$1.5 \%$ (usually a slight underestimation) and a RMS of about $5 \%$ with most of ground-based data sets (Eskes et al., 2005).

\subsection{2 $\mathrm{NO}_{2}$ column}

Validations of the SCIAMACHY $\mathrm{NO}_{2}$ column data were reviewed by Lambert et al. (2004b) in May 2004 for NRT version 5.01 and for scientific products generated at BIRAIASB, IFE/IUP-Bremen, IUP-Heidelberg, KNMI, and SAO. This review was updated with NRT version 5.04 in December 2004. Both NRT versions and the scientific products have demonstrated to capture major geophysical signals appropriately. For NRT 5.01 and 5.04, the two validation reviews conclude to a good agreement (of a few times $10^{14} \mathrm{molec} / \mathrm{cm}^{2}$ ) with correlative data over clean areas in the Southern winterspring and Northern summer. Much larger deviations (systematic overestimations up to $1.5 \cdot 10^{15} \mathrm{molec} / \mathrm{cm}^{2}$ over clean areas and more in polluted conditions) are observed in other cases, e.g. from October till the end of the year for several latitudes but not all, and with a clear correlation with cloud fraction, ghost vertical column and air mass factor values. It is anticipated that, in spring 2005, a GDP 4-like algorithm will be implemented in the OL SCIAMACHY processor and that the $\mathrm{NRT} \mathrm{NO}_{2}$ column product will improve significantly. It is important to note that, while GOME GDP $2.7 \mathrm{NO}_{2}$ columns were strongly affected by instrumental degradation after 2001, the new level-1 calibration implemented in 2002 solved the problem for the subsequent GDP versions, yielding stable data sets over the entire GOME lifetime.

Coordinated validations carried out on the SCIAMACHY $\mathrm{NO}_{2}$ scientific products have shown that stratospheric $\mathrm{NO}_{2}$ columns already have a good quality. Differences between the different products exist because of differences in retrieval settings and assumptions. The retrieval algorithm differences that have most impact concern the profile database used for air mass factor calculations, the way cross-sections temperatures are determined, and the way clouds are handled. More detailed validation is ongoing, with special attention to tropospheric $\mathrm{NO}_{2}$ products. 


\subsubsection{BrO column}

SCIAMACHY BrO slant columns are retrieved by the NRT processor and by scientific algorithms developed at BIRAIASB, IFE/IUP-Bremen, IUP-Heidelberg and SAO. Due to the low signal/noise ratio and to remaining polarisation features of SCIAMACHY in the classical GOME BrO window $(345-359 \mathrm{~nm})$, the SCIAMACHY BrO window in NRT version 5.01 has been shifted to shorter wavelengths (335$347 \mathrm{~nm}$ ). Thanks to this shift, at moderate and large slant column values, an acceptable agreement better than $20 \%$ is found between NRT 5.01 and all scientific SCIAMACHY data sets, and also with GOME despite the spectral window difference. This level of agreement is also observed with ground-based UV-visible measurements after due conversion to vertical columns and appropriate treatment of the diurnal cycle. Detailed investigation further confirms that SCIAMACHY and other BrO sensors capture polar spring emissions and short-term variability in a consistent way. However, in summer, for slant column values smaller than $1.5 \cdot 10^{14} \mathrm{molec} / \mathrm{cm}^{2}$, version 5.01 of the NRT product reports systematically higher values than other systems by $20 \%$ to 100\% (Van Roozendael et al., 2004).

\subsection{4 $\quad \mathrm{SO}_{2}$ column}

SCIAMACHY $\mathrm{SO}_{2}$ column data are routinely retrieved by the scientific processors developed and operated at BIRAIASB and IFE/IUP-Bremen. SCIAMACHY $\mathrm{SO}_{2}$ data give a clear picture of volcanic emissions, sulphur plant fires and other pollution events. They show good agreement with $\mathrm{SO}_{2}$ columns derived from GOME, despite the significant differences in spatial resolution and sampling. Quantitative validation is nevertheless hampered by the lack of independent measurements. Further details are given by Bramstedt et al. (2004).

\subsubsection{OClO slant columns}

SCIAMACHY OClO slant columns produced by the scientific processors at IFE/IUP-Bremen and IUP-Heidelberg have been compared with $\mathrm{OClO}$ slant columns from GOME and with other observations from AMAXDOAS and groundbased UV-visible spectrometers. Using a polarisation sensitivity spectrum and an empirical correction spectrum in the spectral fit, and considering diurnal cycle and atmospheric variability effects, SCIAMACHY OClO data are consistent with those from other sensors and show the expected relation to atmospheric conditions (Wagner et al., 2004; Wang et al., 2003).

\subsection{6 $\mathrm{H}_{2} \mathrm{O}$ columns}

An overview of the validation status of the scientific SCIAMACHY $\mathrm{H}_{2} \mathrm{O}$ columns (IFE/IUP-Bremen, MPI) in May 2004 was given by Timmermans et al. (2004). With the MPI algorithm (based on the GOME algorithm by Lang et al., 2003), a negative bias of 20 to $25 \%$ was reported. The IFE product (Noël et al., 2004) showed about $10 \%$ too low water vapour values when compared to ozone sondes. A more recent validation effort of an improved version of the IFE product shows a systematic bias of $-0.05 \mathrm{~g} / \mathrm{cm}^{2}$, and a scatter of $0.5 \mathrm{~g} / \mathrm{cm}^{2}$ with respect to SSM/I measurements (Noël et al., 2005).

\subsubsection{Clouds}

An overview of the validation status of the SCIAMACHY cloud products in May 2004 was given by Fournier et al. (2004). They concluded that the operational SCIAMACHY cloud fraction correlated well with the cloud fraction retrieved with FRESCO (KNMI), and is not sensitive to errors in the radiance calibration, as FRESCO is. A viewing angle dependent error was pointed out in the operational product. Independent validation still has to be performed. The FRESCO cloud-top pressure shows a RMS difference of about $100 \mathrm{hPa}$ when compared to MODIS (Fournier et al., 2005).

\subsubsection{Aerosol parameters}

An overview of the validation status of the SCIAMACHY aerosol products can be found in von Hoyningen-Hüne et al. (2004) and de Graaf et al. (2004). The aerosol algorithms are very sensitive to errors in the reflectance. However, after using an empirical correction factor for the reflectance, it was shown that the SCIAMACHY Absorbing Aerosol Index (KNMI) compared well to TOMS and the SCIAMACHY Aerosol Optical Thickness (IFE/IUP-Bremen) compared reasonably well to MERIS.

\subsection{Level 2 products from nadir near infrared}

Columns of $\mathrm{CO}, \mathrm{CH}_{4}, \mathrm{~N}_{2} \mathrm{O}$, and/or $\mathrm{CO}_{2}$ have been retrieved from SCIAMACHY near-infrared (NIR) measurements by the IMAP-DOAS (Frankenberg et al., 2005a,b), IMLM (Gloudemans et al., 2005) and WFM-DOAS (Buchwitz et al., 2005b) scientific algorithms. First order corrections for the thermal variation of the dark current signal along the orbit, dead/bad pixels, light leak in channel 7, and the build-up of ice on the detectors of channels 7 and 8 affecting signal transmission and line shape functions, have been implemented. Such corrections significantly improve the level 2 data products, however, the retrieval residuals are still larger than the expected instrument noise. Refinements of the algorithms are still progressing, e.g. using more $\mathrm{CO}$ lines in the retrieval is envisaged. Accurate validation methods are also under refinement, e.g., to deal properly with issues posed by clouds and by small-scale variations of the surface properties and altitude.

Correlative studies have been conducted using groundbased data from a pole-to-pole network of 12 FTIR 
instruments and from the FTIR operated during two cruises of the Polarstern ship from Bremerhaven to Africa; CO column data from the EOS-Terra MOPITT satellite; $\mathrm{CO}$ and $\mathrm{CH}_{4}$ data from the TM3 (KNMI) and TM5 (IMAU) models; and ancillary data like fire maps produced by ERS-2 ATSR and EOS-Aqua MODIS. The general potential of SCIAMACHY NIR products is demonstrated (Buchwitz et al., 2005a; De Mazière et al., 2004; Gloudemans et al., 2005; Warneke et al., 2004; Dils et al., 2005; Sussmann et al., 2005), in particular its capabilities to detect source/sink areas of $\mathrm{CO}, \mathrm{CH}_{4}$ and $\mathrm{CO}_{2}$ and to track their transport. Provisional precision estimates for SCIAMACHY CO $(30 \%)$ and $\mathrm{CH}_{4}(5 \%)$ vertical columns are not far away from the nominal requirements and can already be used in a variety of applications. Even so, inverse modelling analyses seem to indicate that nominal precision requirements are a firm precondition to the potential improvement of existing emission catalogues. The current estimated precision for $\mathrm{N}_{2} \mathrm{O}$ is $20 \%$. It is expected that this will be improved in the near future. For $\mathrm{CO}_{2}$, current validation is too limited to give firm conclusions.

\subsection{Level 2 products retrieved from limb UV-visible}

\subsection{1 $\mathrm{O}_{3}$ profile}

Up to now, the availability of $\mathrm{O}_{3}$ profile data generated from SCIAMACHY limb spectra by the OL processor has been limited to a few verification orbits not designed for geophysical validation, and to a limited data set produced with version 2.1 of the OL processor. Both versions are based on the Optimal Estimation retrieval method. They rely on limb spectra not corrected for pointing errors of the satellite platform and therefore resulting $\mathrm{O}_{3}$ profiles are expected to exhibit a shift in altitude registration. In May 2004, a review of preliminary validations of SCIAMACHY OL $\mathrm{O}_{3}$ profiles (Brinksma et al., 2004) concluded that version $2.1 \mathrm{O}_{3}$ profiles agree to within $10 \%$ to ground-based data (ozone sonde, lidar, microwave, FTIR), and to within $30 \%$ to satellite data (HALOE, SAGE-II, SAGE-III, SBUV/2). More recently, a preliminary validation of version $2.5 \mathrm{O}_{3}$ profiles with ground-based instruments and satellites showed that $20 \%$ of the profiles have unrealistic values. The other $80 \%$ show no systematic deviations above $24 \mathrm{~km}$, but are significantly underestimated below $24 \mathrm{~km}$, by $15 \%$ (Brinksma et al., 2005). It is anticipated that a new $\mathrm{OL}$ limb $\mathrm{O}_{3}$ profile data product based on an alternative retrieval approach and including a correction for pointing errors should be made available to validation teams in 2005 .

Synergistic use of complementary ground-based network data by De Clercq et al. (2004) allowed characterisation of an altitude shift in the ozone profiles. This shift was found to drift monotonically from $1.5 \mathrm{~km}$ to $3 \mathrm{~km}$ between two successive updates of the on-board orbit-model, performed each time Envisat flies over the Caribbean and over Australia. This drift causes fictitious meridional and zonal structures in the $\mathrm{O}_{3}$ profile product. The implementation of a new version of the orbit propagator model on 9 December 2003 improve the altitude registration of the SCIAMACHY O 3 profile (von Savigny et al., 2005).

SCIAMACHY limb $\mathrm{O}_{3}$ profiles have also been generated by IFE/IUP-Bremen. Validation results have been reported for three different software versions: 1.6, 1.61, and 1.62. A cross comparison with GOMOS, MIPAS and SCIAMACHY IFE $1.6 \mathrm{O}_{3}$ profiles showed an agreement within $15 \%$ between 21 and $40 \mathrm{~km}$ (Bracher et al., 2005b). This same version 1.6 agrees within $10-15 \%$ with sondes after an altitude correction of $-2 \mathrm{~km}$ had been applied to account for Envisat pointing problems (Segers et al., 2005). The profiles generated with software version 1.61 were validated for five months spread over 2004 with ground-based and satellite data. The systematic bias of the IFE profiles, after a downward shift of $1.5 \mathrm{~km}$ was applied, is $-3 \%$ with respect to lidars, averaged between 16 and $40 \mathrm{~km}$, and $-6 \%$ with respect to SAGE II over the same latitude range. The difference profile has a characteristic zigzag shape with a wavelength of approximately $8 \mathrm{~km}$, the systematic bias being a few percent larger around 32 and $24 \mathrm{~km}$, and a few percent smaller around 20 and $28 \mathrm{~km}$ (Brinksma et al., 2005). Butz et al. (2005) recently compared balloon borne measurements with SCIAMACHY IFE $1.62 \mathrm{O}_{3}$ profiles. In this software version the tangent height is retrieved explicitly, in order to reduce errors caused by the satellite pointing mismatch. They find an agreement within $20 \%$ between 20 and $30 \mathrm{~km}$. The agreement generally becomes worse below $20 \mathrm{~km}$.

\subsection{2 $\mathrm{NO}_{2}$ profiles}

An overview of the validation status of the SCIAMACHY $\mathrm{NO}_{2}$ profiles (OL vs. 2.1) in May 2004 was given by von Savigny et al. (2004). They concluded that the OL product was about $50 \%$ larger than HALOE between 25 and $40 \mathrm{~km}$ (Bracher et al., 2005c). About $10 \%$ of the OL profiles appear to be entirely unrealistic. The IFE/IUP-Bremen profiles were within $15 \%$ of the HALOE profiles $(22-33 \mathrm{~km})$. Both IFE and $\mathrm{SAO} \mathrm{NO}_{2}$ profiles compared reasonably to $\mathrm{SAOZ}$ balloon profiles.

A more recent comparison of OL vs. 2.4 and $2.5 \mathrm{NO}_{2}$ profiles with collocated and photochemically corrected SAGE II (vs. 6.2) measurements yield improved $\mathrm{OL} \mathrm{NO}_{2}$ profiles (Bracher, personal communication, Feb. 2005). However, the quality of the retrievals strongly depend on latitude and/or solar zenith angle (SZA). For high southern latitudes $\left(60^{\circ} \mathrm{S}-\right.$ $\left.70^{\circ} \mathrm{S}\right)$ the $\mathrm{OL} \mathrm{NO}_{2}$ profile retrievals agree with photochemically corrected SAGE II profiles within $-20 \%$ to $+5 \%$ for the $19-36 \mathrm{~km}$ altitude range. At low northern latitudes $\left(25^{\circ} \mathrm{N}\right.$ $30^{\circ} \mathrm{N}$ ) the $\mathrm{OL}$ concentrations were systematically higher by $10-60 \%$ in the $25-36 \mathrm{~km}$ altitude range. At northern mid-latitudes $\left(40^{\circ} \mathrm{N}-53^{\circ} \mathrm{N}\right)$ the agreement was again better: $-35 \%$ to $+25 \%$ between 19 and $36 \mathrm{~km}$. 
Retrievals with the DLR prototype processor that will replace the current OL processor in the future are in good agreement with the IFE $\mathrm{NO}_{2}$ profile retrievals (Doicu et al., $2006^{1}$ ).

Comparisons between SCIAMACHY $\mathrm{NO}_{2}$ profiles retrieved by IFE/IUP-Bremen (version 1) and HALOE (version 19) show good agreement (within 12\%) between 22 and $33 \mathrm{~km}$. Comparisons with SAGE II show a systematic negative bias of 10 to $35 \%$ between 20 and $38 \mathrm{~km}$, where SAGE II sunset profiles are suspected to be too high (Bracher et al., 2005c). A cross comparison with GOMOS, MIPAS and SCIAMACHY IFE v1 $\mathrm{NO}_{2}$ profiles show an agreement within $20 \%$ between 27 and $40 \mathrm{~km}$ (Bracher et al., 2005b). Butz et al. (2005) recently compared balloon borne measurements with SCIAMACHY NO 2 profiles retrieved by IFE/IUP-Bremen, IUP-Heidelberg, and SAO Harvard. They find an agreement within $20 \%$ between 20 and $30 \mathrm{~km}$. The agreement generally becomes worse below $20 \mathrm{~km}$.

\subsubsection{BrO profiles}

Profiles of BrO have been retrieved from SCIAMACHY limb spectra by IFE/IUP-Bremen, SAO, and IUP-Heidelberg. Preliminary validation relies on comparisons with balloon-based data acquired either by UV-visible solar occultation during ascent/descent and sunset/sunrise (three LPMA/DOAS and seven SAOZ-balloon flights) or by resonance fluorescence (three TRIPLE flights), and with simulations provided by the SLIMCAT chemical-transport model of the stratosphere. The direct comparison of photochemically uncorrected $\mathrm{BrO}$ profiles from balloons and from SCIAMACHY limb spectra shows promising results in the middle stratosphere but reveals possible problems below $20 \mathrm{~km}$ (Dorf et al., 2005; Pfeilsticker et al., 2004; Rozanov et al., 2005). Further validation is needed, that will include photochemical corrections for balloon observations along calculated air mass trajectories.

\section{Validation plans}

Scientific publications in which SCIAMACHY products are used need to address the quality (like accuracy, precision, features) of these products focused on the effect the quality has on the results presented in the publication. It is our goal to give a general description of the quality for all SCIAMACHY products that are well-documented and generally available, including both operational and scientific products.

A complete description of the quality of a SCIAMACHY product should be formulated in such a way that it is of direct use for scientific research. In particular the behaviour

\footnotetext{
${ }^{1}$ Doicu, A., Hilgers, S., Rozanov, A., Eichmann, K.-U., von Savigny, Ch., and Burrows, J. P.: Information operator approach for atmospheric remote sensing, J. Quant. Spectrosc. Radiat. Transfer, submitted, 2006.
}

of deviations (between SCIAMACHY data and correlative data) with respect to relevant algorithm, instrument, and atmospheric parameters should be specified. Examples of such parameters are: surface albedo, scan mirror angle, solar zenith angle. Some product specific features (or obvious errors) can be observed from the analysis of the product itself, without comparison to correlative data. Also, the descriptions of these features, and where they occur (what algorithm, instrumental or geophysical parameters), should be part of the complete description.

Ideally, all SCIAMACHY products should be compared to several, i.e. more than one, independent sources of wellestablished correlative data. The results of these comparisons should be combined to get a consistent description of the behaviour of the product.

To achieve our long-term goal it is necessary that: 1) processors are bug free and known improvements to the algorithms are implemented, 2) a consecutive period of at least one year of data is processed with the latest software version, and enough collocations with correlative data are included within this data set, and 3) the manpower for data analysis is available.

The current operational nadir UV-visible products (NRT 5.04 and $\mathrm{OL} 2.5$ ), i.e., vertical columns of $\mathrm{O}_{3}$ and $\mathrm{NO}_{2}$, and slant columns of $\mathrm{BrO}, \mathrm{OClO}$ and $\mathrm{HCHO}$, are based on the algorithms used in the GOME Data Processor (GDP) version 2.4. An upgrade of these algorithms to the algorithms currently used in GOME (GDP version 4.0) is currently being implemented and reprocessed data should be available in early 2006. Validation of the current product has confirmed similar features of $\mathrm{O}_{3}$ and $\mathrm{NO}_{2}$ as in GOME version 2.4 (see Sects. 6.2.1 and 6.2.2), and further validation is waiting for the processor upgrade.

The current operational nadir NIR products are still unrealistic, see Sect. 6.3. Upgrades of the level $0-1$ and the level 1-2 processors should improve these products; the validation can hopefully start after these upgrades have been performed. For NIR products this will not be done before 2006 .

In the $\mathrm{O}_{3}$ and $\mathrm{NO}_{2}$ profiles, generated with the operational OL processor version 2.5, several unrealistic features have been found (see Sects. 6.4.1 and 6.4.2). Validation of these products should also continue after the next processor upgrade, planned for fall 2005.

For many of the products of SCIAMACHY enough correlative measurements are available. However, the SCIAMACHY measurements themselves are not all processed with the current operational processors (NRT 5.04/OL 2.5) and/or distributed to the validation teams, making it difficult to continue or complete the validation of the operational products. To monitor the long-term product quality of the instrument, a basic amount of correlative data over SCIAMACHY's lifetime is required.

Most of the available funding for SCIAMACHY validation from the national space agencies of Germany, The Netherlands, and Belgium, was applied in the first two years 
of operation, since the necessary effort was expected to be largest in that period. Dedicated validation measurements have indeed been performed successfully, but the analysis of the SCIAMACHY data still has to be performed for all SCIAMACHY products after bugs have been fixed, algorithms have been improved, and the data have been reprocessed and distributed. Only very limited funding is currently available to perform these tasks.

\section{Conclusions}

With the support of ESA and of a list of international partners, an extensive SCIAMACHY validation programme has been developed jointly by Germany, the Netherlands and Belgium to face complex requirements in terms of measured species, altitude range, spatial and temporal scales, geophysical states and intended applications. Preparation of the validation has been successful:

- An organisational structure has been set up to coordinate this large-scale validation campaign, to monitor continuously the validation results (Sect. 3) and to foster exchanges between the different validation actors;

- Numerous independent validation measurements of all planned SCIAMACHY products have been performed (Sect. 4), with a special concentration in the first two years of operation;

- The required manpower to analyse the data was available in the first two years of operation, for a large part funded by the national space agencies in the three instrument-providing countries.

The validation itself has been however hampered, firstly by delays in the production and delivery of operational data by the Envisat ground segment, secondly by delays in the development of the data processors.

Since the first release of early SCIAMACHY data in summer 2002, the operational processors established at DLR on behalf of ESA were upgraded regularly (see Table 5) and some data products (level 1b spectra, $\mathrm{O}_{3}, \mathrm{NO}_{2}, \mathrm{BrO}$ and clouds data) have improved considerably. Validation has shown that for limited periods and geographical domains they can already be used for scientific studies. Nevertheless, the NRT level-1-to-2 processor is still affected by a series of bugs, which result in major errors preventing from scientific usability in other periods and domains, e.g., the $\mathrm{O}_{3}$ and $\mathrm{NO}_{2}$ columns in the last months of the year or the $\mathrm{BrO}$ columns at small $\mathrm{BrO}$ abundances. Scientific users are advised to read carefully validation reports and quality statements before using the data.

Free from the constraints of operational processing, independent research algorithms developed at scientific institutes usually are more flexible and they allow dedicated investigation of specific retrieval issues. Seven scientific institutes (BIRA-IASB, IFE/IUP-Bremen, IUP-Heidelberg, KNMI, MPI, SAO and SRON) have developed their own retrieval algorithms and generated SCIAMACHY data products, addressing all together nearly all targeted constituents. Their UV-visible data products $-\mathrm{O}_{3}, \mathrm{NO}_{2}, \mathrm{SO}_{2}, \mathrm{H}_{2} \mathrm{O}$ total columns; $\mathrm{BrO}, \mathrm{OClO}$ slant columns; $\mathrm{O}_{3}, \mathrm{NO}_{2}, \mathrm{BrO}$ profiles are already of acceptable, if not of excellent, quality and are available publicly, sometimes in near-real-time (see Table 1 and Sect. 6). Several near-infrared column products - CO, $\mathrm{CH}_{4}, \mathrm{~N}_{2} \mathrm{O}$ and $\mathrm{CO}_{2}$ - are still under development but they have already demonstrated their potential for a variety of applications. Most of them can be obtained on request. All scientific products will be judged first on their availability and description and after that on their usefulness for scientific research by the monitoring of the validation results.

SCIAMACHY is a remarkable stable instrument starting the fourth year of almost continous measurements (see Fig. 1). It is required and anticipated that SCIAMACHY validation will continue throughout the instrument lifetime and beyond, anticipating algorithm updates and reprocessing of data for many years to come. The actual amount of work will obviously depend on funding and manpower considerations.

Acknowledgements. SCIAMACHY is a national contribution to the Envisat Mission of the European Space Agency (ESA). It is funded by the German Aerospace Center (DLR) and the Netherlands Agency for Aerospace Programs (NIVR) with a contribution of the Belgian Institute for Space Aeronomy (BIRA-IASB). We thank the agencies for providing the platform and the instrument. SCIAMACHY's Principal Investigator is J. Burrows (University of Bremen) and Co-Principal Investigators are A. Goede (KNMI) and C. Muller (BIRA-IASB). Operational data processing is performed at the German Remote Sensing Data Center (DLR-DFD) on behalf of ESA as part of the Envisat ground segment.

Validation activities are initiated by the Principal Investigators of the ESA-AO, by alphabetical order: I. Aben, C. Blom, T. Blumenstock, J. Burrows, C. Camy-Peyret, K. Chance, W. Chu, P. Ciotti, F. Congeduti, V. Cuomo, O. F. d'Andon, M. De Maziere, D. De Muer, C. Deniel, M. Dobber, H. Fischer, D. Fonteyn, K.-H. Fricke, F. Goutail, J. Haase, G. Hansen, E. Hilsenrath, A. Hollingsworth, P. Keckhut, H. Kelder, E. Kyrölä, K. Labitzke, W. Lahoz, J.-C. Lambert, A. Matthews, C. Matzler, D. Murtagh, S. Pal, P. Peeters, K. Pfeilsticker, M. Pirre, J.-P. Pommereau, R. Rood, P. Stammes, L. Stefanutti, D. Swart, J. Tamminen, Y. Timofeyev, and M. Weber. We thank them and their co-workers warmly for providing correlative data and for carrying out validation studies. Part of the correlative data was obtained in the framework of WMO's Global Atmospheric Watch Programme (GAW) and is publicly available via the NDSC and WOUDC archives (see http://www.ndsc.ws and http://www.woudc.org).

The good interaction and cooperation with ESA on validation issues has been greatly appreciated. We thank especially J. Frerick, R. Koopman, P. Snoeij, C. Zehner, P. Wursteisen, T. Wehr, A. Dehn, and E. Attema.

This paper is for a major part based on the discussions and cooperation within the SCIAVALIG team. We thank the other members: H. Kelder, U. Platt, P. Simon, R. Timmermans, I. Aben, J. Burrows, 
C. Camy-Peyret, E. Hilsenrath, B. Kerridge, K. Künzi, D. Perner, M. Riese, H. Smit, J. Staehelin, and D. Swart.

The SCIAMACHY validation product coordinators are acknowledged for their support in summarising the validation results: G. Lichtenberg, G. Tilstra, A. Richter, M. van Roozendael, T. Wagner, M. de Mazière, C. Camy-Peyret, J. Notholt, W. von HoyningenHüne, F. Wittrock, R. Timmermans, M. van Weele, and B. Barret.

SCIAMACHY Validation has been funded partly by the European Space Agency, ProDEx, the Science Policy Office of the Belgian Prime Minister's Services, the Bundesministerium für Bildung und Forschung (BMBF via DLR-Bonn, Germany, Projects 55EE 9908/09 and 50EE0005-0025), the Netherlands Agency for Aerospace Programmes (NIVR), and the French Programme National de Chimie de l'Atmosphère (PNCA).

Edited by: P. Monks

\section{References}

Acarreta, J. R. and Stammes, P.: Calibration comparison between SCIAMACHY and MERIS on board ENVISAT, IEEE Geoscience and Remote Sensing Letters, 2, 1, 31-35, 2005.

Bovensmann, H., Burrows, J. P., Buchwitz, M., Frerick, J., Noël, S., Rozanov, V. V., Chance, K. V., and Goede, A. P. H.: SCIAMACHY: Mission objectives and measurement modes, J. Atmos. Sci., 56, 2, 127-150, 1999.

Bracher, A., Weber, M., Bramstedt, K., Coldewey-Egbers, M., Lamsal, L., and Burrows, J. P.: Global satellite validation of SCIAMACHY $\mathrm{O}_{3}$ columns with GOME WFDOAS, Atmos. Chem. Phys., 5, 2357-2368, 2005a,

SRef-ID: 1680-7324/acp/2005-5-2357.

Bracher, A., Bovensmann, H., Bramstedt, K., Burrows, J.P., von Clarmann, T., Eichmann, K.-U., Fischer, H., Funke, B., GilLópez, S., Glatthor, N., Grabowski, U., Höpfner, M., Kaufmann, M., Kellmann, S., Kiefer, M., Koukouli, M. E., Linden, A., López-Puertas, M., Mengistu Tsidu, G., Milz, M., Noël, S., Rohen, G., Rozanov, A., Rozanov, V. V., von Savigny, C., Skupin, J., Sinnhuber, M., Steck, T., Stiller, G. P., Wang, D.-Y., Weber, M., and Wuttke, M. W.: Cross comparisons of $\mathrm{O}_{3}$ and $\mathrm{NO}_{2}$ profiles measured by the atmospheric ENVISAT instruments GOMOS, MIPAS, and SCIAMACHY, Adv. Space Res., 36, 5, 855867, 2005b.

Bracher, A., Sinnhuber, M., Rozanov, A., and Burrows, J. P.: Using a photochemical model for the validation of $\mathrm{NO}_{2}$ satellite measurements at different solar zenith angles, Atmos. Chem. Phys., 5, 393-408, 2005c,

\section{SRef-ID: 1680-7324/acp/2005-5-393.}

Bramstedt, K., Richter, A., Van Roozendael, M., and De Smedt, I.: Comparisons of SCIAMACHY sulfur dioxide observations, Proceedings of the Second Workshop on the Atmospheric Validation of Envisat (ACVE-2), 3-7 May 2004, Frascati, ESA SP562,2004

Brinksma, E. J., Piters, A. J. M., Boyd, I. S., Parrish, A., Bracher, A., von Savigny, C., Bramstedt, K., Schmoltner, A.-M., Taha, G., Hilsenrath, E., Blumenstock, T., Kopp, G., Mikuteit, S., Fix, A., Meijer, Y. J., Swart, D. P. J., Bodeker, G. E., McDermid, I. S., and Leblanc, T.: SCIAMACHY ozone profile validation, Proceed- ings of the Second Workshop on the Atmospheric Validation of Envisat (ACVE-2), 3-7 May 2004, Frascati, ESA SP-562, 2004.

Brinksma, E. J., Bracher, A., Lolkema, D. E., et al.: Geophysical validation of SCIAMACHY limb ozone profiles, Atmos. Chem. Phys. Discuss., 5, 4893-4928, 2005,

SRef-ID: 1680-7375/acpd/2005-5-4893.

Buchwitz, M., de Beek, R., Burrows, J. P., Bovensmann, H., Warneke, T., Notholt, J., Meirink, J. F., Goede, A. P. H., Bergamaschi, P., Körner, S., Heimann, M., and Schulz, A.: Atmospheric methane and carbon dioxide from SCIAMACHY satellite data: initial comparison with chemistry and transport models, Atmos. Chem. Phys., 5, 941-962, 2005a,

SRef-ID: 1680-7324/acp/2005-5-941.

Buchwitz, M., de Beek, R., Noël, S., Burrows, J. P., Bovensmann, H., Bremer, H., Bergamaschi, P., Körner, S., and Heimann, M.: Carbon monoxide, methane and carbon dioxide columns retrieved from SCIAMACHY by WFM-DOAS: Year 2003 initial data set, Atmos. Chem. Phys., 5, 3313-3329, 2005b,

SRef-ID: 1680-7324/acp/2005-5-3313.

Butz, A., Bösch, H., Camy-Peyret, C., Chipperfield, M., Dorf, M., Dufour, G., Grunow, K., Jeseck, P., Kühl, S., Payan, S., Pepin, I., Pukite, J., Rozanov, A., von Savigny, C., Sioris, C., Wagner, T., Weidner, F., and Pfeilsticker, K.: Inter-comparison of stratospheric $\mathrm{O}_{3}$ and $\mathrm{NO}_{2}$ abundances retrieved from balloon borne direct sun observations and Envisat/SCIAMACHY limb measurements, Atmos. Chem. Phys. Discuss., 5, 10747-10 797, 2005, SRef-ID: 1680-7375/acpd/2005-5-10747.

Chlebek, Ch., Noël, S., Skupin, J., Gottwald, M., and Krieg, E.: SCIAMACHY instrument status, Proceedings of the Second Workshop on the Atmospheric Validation of Envisat (ACVE-2), 3-7 May 2004, Frascati, ESA SP-562, 2004.

Coldewey-Egbers, M., Weber, M., Lamsal, L. N., de Beek, R., Buchwitz, M., and Burrows, J. P.: Total ozone retrieval from GOME UV spectral data using the weighting function DOAS approach, Atmos. Chem. Phys., 5, 1015-1025, 2005,

SRef-ID: 1680-7324/acp/2005-5-1015.

De Clercq, C., Lambert, J.-C., Calisesi, Y., Claude, H., Stubi, R., von Savigny, C., and the ACVT-GBMCD Ozone Profile Team: Integrated characterization of Envisat ozone profile data using ground-based network data, Proceedings of the Envisat and ERS Symposium, Salzburg, Austria, 6-10 September 2004, ESA SP572, 2004.

De Mazière, M., Barret, B., Blumenstock, T., Buchwitz, M., de Beek, R., Demoulin, P., Fast, H., Gloudemans, A., Griesfeller, A., Griffith, D., Ionov, D., Janssens, K., Jones, N., Mahieu, E., Mellqvist, J., Mittermeier, R. L., Notholt, J., Rinsland, C., Schrijver, H., Schultz, A., Smale, D., Strandberg, A., Strong, K., Sussmann, R., Warneke, T., and Wood, S.: Comparisons between SCIAMACHY scientific products and ground-based FTIR data for total columns of $\mathrm{CO}, \mathrm{CH}_{4}$ and $\mathrm{N}_{2} \mathrm{O}$, Proceedings of the Second Workshop on the Atmospheric Validation of Envisat (ACVE2), 3-7 May 2004, Frascati, ESA SP-562, 2004.

Dils, B., De Mazière, M., Blumenstock, T., Buchwitz, M., de Beek, R., Demoulin, P., Duchatelet, P., Fast, H., Frankenberg, C., Gloudemans, A., Griffith, D., Jones, N., Kerzenmacher, T., Kramer, I., Mahieu, E., Mellqvist, J., Mittermeier, R. L., Notholt, J., Rinsland, C. P., Schrijver, H., Smale, D., Strandberg, A., Straume, A. G., Stremme, W., Strong, K., Sussmann, R., Taylor, J., van den Broek, M., Wagner, T., Warneke, T., Wiacek, A., 
and Wood, S.: Comparisons between SCIAMACHY and groundbased FTIR data for total columns of $\mathrm{CO}, \mathrm{CH}_{4}, \mathrm{CO}_{2}$ and $\mathrm{N}_{2} \mathrm{O}$, Atmos. Chem. Phys. Discuss., 5, 2677-2717, 2005, SRef-ID: 1680-7375/acpd/2005-5-2677.

Dorf, M., Camy-Peyret, C., Chipperfield, M., Engel, A., Goutail, F., Grunow, K., Van Roozendael, M., Sioris, C., Stroh, F., and Pfeilsticker, K.: Balloon-borne stratospheric BrO measurements: Comparison with Envisat/SCIAMACHY BrO limb profiles, Atmos. Chem. Phys. Discuss., 5, 13 011-13 052, 2005,

SRef-ID: 1680-7375/acpd/2005-5-13011.

Eskes, H. J., van der A. R. J., Brinksma, E. J., Veefkinf, J. P., de Haan, J. F., and Valks, P. J. M.: Retrieval and validation of ozone columns derived from measurements of SCIAMACHY on Envisat, Atmos. Chem. Phys. Discuss., 5, 4429-4475, 2005,

SRef-ID: 1680-7375/acpd/2005-5-4429.

Fioletov, E., Kerr, J. B., Hare, E. W., Labow, G. J., and McPeters, R. D.: An assessment of the world ground-based total ozone network performance from the comparison with satellite data, J. Geophys. Res., 104(1), 1737-1747, 1999.

Fix, A., Ehret, G., Flentje, H., Poberaj, G., Gottwald, M., Finkenzeller, H., Bremer, H., Bruns, M., Burrows, J. P., Kleinböhl, A., Küllmann, H., Kuttippurath, J., Richter, A., Wang, P., Heue, K.P., Platt, U., and Wagner, T.: SCIAMACHY validation by aircraft remote measurements: design, execution, and first results of the SCIA-VALUE mission, Atmos. Chem. Phys., 5, 12731289, 2005,

SRef-ID: 1680-7324/acp/2005-5-1273

Fournier, N., Stammes, P., Acarreta, J. R., Eskes, H., Piters, A., Hess, M., Von Bargen, A., Kokhanovsky, A., and Grzegorski, M.: SCIAMACHY cloud product validation, Proceedings of the Second Workshop on the Atmospheric Validation of Envisat (ACVE2), 3-7 May 2004, Frascati, ESA SP-562, 2004.

Fournier, N., Stammes, P., de Graaf, M., van der A, R., Piters, A., Koelemeijer, R., and Kokhanovsky, A.: Improving cloud information over deserts from SCIAMACHY O 2 A-band, Atmos. Chem. Phys. Discuss., 5, 6013-6039, 2005,

SRef-ID: 1680-7375/acpd/2005-5-6013.

Frankenberg, C., Platt, U., and Wagner, T.: Retrieval of CO from SCIAMACHY onboard ENVISAT: Detection of strongly polluted areas and seasonal patterns in global $\mathrm{CO}$ abundances, Atmos. Chem. Phys., 5, 1639-1644, 2005a,

SRef-ID: 1680-7324/acp/2005-5-1639.

Frankenberg, C., Platt, U., and Wagner, T.: Iterative maximum a posteriori (IMAP-)DOAS for retrieval of strongly absorbing trace gases: Model studies for $\mathrm{CH}_{4}$ and $\mathrm{CO}_{2}$ retrieval from nearinfrared spectra of SCIAMACHY onboard ENVISAT, Atmos. Chem. Phys., 5, 9-22, 2005b,

SRef-ID: 1680-7324/acp/2005-5-9.

Gloudemans, A. M. S., Schrijver, H., Kleipool, Q., van den Broek, M. M. P., Straume, A. G., Lichtenberg, G., van Hees, R. M., Aben, I., and Meirink, J. F.: The impact of SCIAMACHY nearinfrared instrument calibration on $\mathrm{CH}_{4}$ and $\mathrm{CO}$ total columns, Atmos. Chem. Phys., 5, 2369-2383, 2005,

SRef-ID: 1680-7324/acp/2005-5-2369.

de Graaf, M., Tilstra, L. G., and Stammes, P.: SCIAMACHY Absorbing Aerosol Index: The scientific product compared to the operational product and TOMS data, Proceedings of the Second Workshop on the Atmospheric Validation of Envisat (ACVE-2), 3-7 May 2004, Frascati, ESA SP-562, 2004.
Gurlit, W., Bösch, H., Bovensmann, H., Burrows, J. P., Butz, A., Camy-Peyret, C., Dorf, M., Gerilowski, K., Lindner, A., Noël, S., Platt, U., Weidner, F., and Pfeilsticker, K.: The UV-A and visible solar irradiance spectrum: inter-comparison of absolutely calibrated, spectrally medium resolution solar irradiance spectra from balloon- and satellite-borne measurements, Atmos. Chem. Phys., 5, 1879-1890, 2005,

SRef-ID: 1680-7324/acp/2005-5-1879.

Heland, J., Schlager, H., Schiller, C., Sitnikov, N., Ulanovsky, A., Ravegnani, F., Volk, C. M., Werner, A., Petritoli, A., Kostadinov, I., Giovanelli, G., Bortoli, D., Stroh, F., von Hobe, M., and the Geophysica Team: Validation of MIPAS on Envisat by in situ instruments on the M55-Geophysica, Proceedings of the Envisat Validation Workshop, Frascati, Italy, 9-13 December 2002, ESA SP-531, 2003.

von Hoyningen-Hüne, W.: Validation of aerosol products derived from SCIAMACHY, Proceedings of the Second Workshop on the Atmospheric Validation of Envisat (ACVE-2), 3-7 May 2004, Frascati, ESA SP-562, 2004.

Kelder, H., Piters, A., Timmermans, R., Bramstedt, K., and Lambert, J.-C.: SCIAMACHY validation summary, Proceedings of the Second Workshop on the Atmospheric Validation of Envisat (ACVE-2), 3-7 May 2004, Frascati, ESA SP-562, 2004.

Kostadinov, I., Giovanelli, G., Petritoli, A., Bortoli, D., Ravegnani, F., Radaelli, G., Ulanovsky, A., and Yuzhkov, V.: Combined in situ and quasi in situ measurements aboard M55 Geophysica stratospheric aircraft dedicated for Envisat satellite data validation, Proceedings of the Envisat Validation Workshop, Frascati, Italy, 9-13 December 2002, ESA SP-531, 2003.

Lambert, J.-C., Van Roozendael, M., De Mazière, M., Simon, P. C., Pommereau, J.-P., Goutail, F., Sarkissian, A., and Gleason, J. F.: Investigation of pole-to-pole performances of spaceborne atmospheric chemistry sensors with the NDSC, J. Atmos. Sci., 56, 176-193, 1999.

Lambert, J.-C., Allaart, M., Andersen, S. B., Blumenstock, T., Bodeker, G., Brinksma, E., Cambridge, C., De Mazière, M., Demoulin, P., Gerard, P., Gil, M., Goutail, F., Granville, J., Ionov, D. V., Kyrö, E., Navarro-Comas, M., Piters, A., Pommereau, J.-P., Richter, A., Roscoe, H. K., Schets, H., Shanklin, J. D., Suortti, T., Sussmann, R., Van Roozendael, M., Varotsos, C., Wagner, T., Wood, S., and Yela, M.: First ground-based validation of SCIAMACHY V5.01 ozone column, Proceedings of the Second Workshop on the Atmospheric Validation of Envisat (ACVE-2), 3-7 May 2004, Frascati, Italy, ESA SP-562, 2004a.

Lambert, J.-C., Blumenstock, T., Boersma, F., Bracher, A., De Mazière, M., Demoulin, P., De Smedt, I., Eskes, H., Gil, M., Goutail, F., Granville, J., Hendrick, F., Ionov, D. V., Johnston, P. V., Kostadinov, I., Kreher, K., Kyrö, E., Martin, R., Meier, A., Navarro-Comas, M., Petritoli, A., Pommereau, J.-P., Richter, A., Roscoe, H. K., Sioris, C., Sussmann, R., Van Roozendael, M., Wagner, T., Wood, S., and Yela, M.: Geophysical validation of SCIAMACHY $\mathrm{NO}_{2}$ vertical columns: Overview of early 2004 results, Proceedings of the Second Workshop on the Atmospheric Validation of Envisat (ACVE-2), 3-7 May 2004, Frascati, Italy, ESA SP-562, 2004b.

Lang, R., Williams, J. E., Maurellis, A. N., and van der Zande, W. J.: Application of the Spectral Structure Parameterization Technique: Retrieval of Total Water Vapor Columns from GOME, Atmos. Chem. Phys., 3, 145-160, 2003, 
SRef-ID: 1680-7324/acp/2003-3-145.

Lichtenberg, G., Kleipool, Q., Krijger, J. M., et al.: SCIAMACHY Level1 data: Calibration concept and in-flight calibration, Atmos. Chem. Phys. Discuss., 5, 8925-8977, 2005,

SRef-ID: 1680-7375/acpd/2005-5-8925.

Marenco, A., Thouret, V., Nédélec, P., Smit, H., Helten, M., Kley, D., Karcher, F., Simon, P., Law, K., Pyle, J., Poschmann, G., von Wrede, R., Hume, C., and Cook, T.: Measurement of ozone and water vapor by Airbus in-service aircraft: The MOZAIC airborne program, An overview, J. Geophys. Res., 103, 25 631$25642,1998$.

Noël, S., Buchwitz, M., and Burrows, J. P.: First retrieval of global water vapour column amounts from SCIAMACHY measurements, Atmos. Chem. Phys., 4, 111-125, 2004,

SRef-ID: 1680-7324/acp/2004-4-111.

Noël, S., Buchwitz, M., Bovensmann, H., and Burrows, J. P.: Validation of SCIAMACHY AMC-DOAS water vapour columns, Atmos. Chem. Phys., 5, 1835-1841, 2005,

SRef-ID: 1680-7324/acp/2005-5-1835

Pfeilsticker, K., Bösch, H., Burrows, J., Butz, A., Camy-Peyret, C., Dorf, M., Goutail, F., Grunow, K., Hrechanyy, S., Naujokat, B., Pommereau, J. P., Sioris, C., Stroh, F., Rozanov, A., and Weidner, F.: Validation of SCIAMACHY BrO Profiles, Proceedings of the Second Workshop on the Atmospheric Validation of Envisat (ACVE-2), 3-7 May 2004, Frascati, ESA SP-562, 2004.

Rozanov, A., Bovensmann, H., Bracher, A., Hrechanyy, S., Rozanov, V., Sinnhuber, M., Stroh, F., and Burrows, J. P.: $\mathrm{NO}_{2}$ and $\mathrm{BrO}$ vertical profile retrieval from SCIAMACHY limb measurements: sensitivity studies, Adv. Space Res., 36, 5, 846-854, 2005.

von Savigny, C., Bracher, A., Bramstedt, K., Rozanov, A., Sinnhuber, M., Sioris, C., Butz, A., Dorf, M., Pfeilsticker, K., Grunow, K., Goutail, F., Pommereau, J. P., and Huret, N.: SCIAMACHY limb $\mathrm{NO}_{2}$ profile validation, Proceedings of the Second Workshop on the Atmospheric Validation of Envisat (ACVE-2), 3-7 May 2004, Frascati, ESA SP-562, 2004.

von Savigny, C., Kaiser, J. W., Bovensmann, H., Burrows, J. P., McDermid, I. S., and Leblanc, T.: Spatial and temporal characterization of SCIAMACHY limb pointing errors during the first three years of the mission, Atmos. Chem. Phys., 5, 2593-2602, 2005 ,

SRef-ID: 1680-7324/acp/2005-5-2593.

SCIAVALIG: SCIAMACHY Validation Requirements, SVDS-01, published by KNMI and NIVR, January 1998.

SCIAVALIG: SCIAMACHY Detailed Validation Plan, SVDS-04, published by KNMI and NIVR, October 2002.

Segers, A. J., von Savigny, C., Brinksma, E. J., and Piters, A. J. M.: Validation of IFE-1.6 SCIAMACHY limb ozone profiles, Atmos. Chem. Phys., 5, 3045-3052, 2005,

SRef-ID: 1680-7324/acp/2005-5-3045

Skupin, J., Noël, S., Wuttke, M. W., Gottwald, M., Bovensmann, H., Weber, M., and Burrows, J. P.: SCIAMACHY Solar Irradiance Observation in the Spectral Range from 240 to $2380 \mathrm{~nm}$, Adv. Space Res., 35, 3, 370-375, 2005. van Soest, G., Tilstra, L. G., and Stammes, P.: Large-scale validation of SCIAMACHY reflectance in the ultraviolet, Atmos. Chem. Phys., 5, 2171-2180, 2005,

SRef-ID: 1680-7324/acp/2005-5-2171.

Spurr, R. J. D., van Roozendael, M., and Loyola, D. G.: Algorithm Theoretical Basis Document for GOME Total Column Densities of Ozone and Nitrogen Dioxide UPAS/GDOAS: GDP 4.0, DLR Technical note ERSE-DTEX-EOPG-TN-04-0007, issue 1A, DLR, Oberpfaffenhofen, Germany, December 2004.

Sussmann, R., Stremme, W., Buchwitz, M., and de Beek, R.: Validation of ENVISAT/SCIAMACHY columnar methane by solar FTIR spectrometry at the Ground-Truthing Station Zugspitze, Atmos. Chem. Phys., 5, 2419-2429, 2005,

SRef-ID: 1680-7324/acp/2005-5-2419.

Tilstra, L. G., van Soest, G., de Graaf, M., Acarreta, J. R., and Stammes, P.: Reflectance comparison between SCIAMACHY and a radiative transfer code in the UV, Proceedings of the Second Workshop on the Atmospheric Validation of Envisat (ACVE2), 3-7 May 2004, Frascati, ESA SP-562, 2004.

Tilstra, L. G. and Stammes, P.: Alternative polarisation retrieval for SCIAMACHY in the ultraviolet, Atmos. Chem. Phys., 5, 2099 2107, 2005,

SRef-ID: 1680-7324/acp/2005-5-2099.

Timmermans, R. M. A., Lang, R., Noël, S., Kois, B., and Kyrö, E.: SCIAMACHY $\mathrm{H}_{2} \mathrm{O}$ column validation by the atmospheric chemistry validation team, Proceedings of the Second Workshop on the Atmospheric Validation of Envisat (ACVE-2), 3-7 May 2004, Frascati, ESA SP-562, 2004.

Valks, P. and van Oss, R.: TOGOMI Algorithm Theoretical Basis Document, Issue 1.2, TOGOMI/KNMI/ATBD/001, KNMI/ESA, November 2003.

Van Roozendael, M., De Smedt, I., Fayt, C., Wittrock, F., Richter, A., and Afe, O.: First validation of SCIAMACHY BrO columns, Proceedings of the Second Workshop on the Atmospheric Validation of Envisat (ACVE-2), 3-7 May 2004, Frascati, ESA SP$562,2004$.

Wagner, T., Kühl, S., Richter, A., Bruns, M., Burrows, J. P., Heue, K.-P., Kirchoff, B., Wilms-Grabe, W., Wang, P., and Platt, U.: Preliminary validation of SCIAMACHY nadir OClO SCDS, Proceedings of the Second Workshop on the Atmospheric Validation of Envisat (ACVE-2), 3-7 May 2004, Frascati, ESA SP-562, 2004.

Wang, P., Richter, A., Bruns, M., Burrows, J. P., Heue, K.-P., Wagner, T., Pundt, I., and Platt, U.: AMAXDOAS OClO measurements in EUPLEX campaign, Proceedings of the 16th ESA Symposium on on rocket and balloon program and related research, 2-5 June 2003, St. Gallen, ESA SP-530, 2003.

Warneke, T., de Beek, R., Buchwitz, M., Notholt, J., Schulz, A., Velazco, V., and Schrems, O.: Shipborne solar absorption measurements of $\mathrm{CO}_{2}, \mathrm{CH}_{4}, \mathrm{~N}_{2} \mathrm{O}$ and $\mathrm{CO}$ and comparison with SCIAMACHY WFM-DOAS retrievals, Atmos. Chem. Phys., 5, 20292034, 2005,

SRef-ID: 1680-7324/acp/2005-5-2029. 